\title{
Development and Validation of SPECT Radionuclide Angiography Phase Analysis Techniques for Quantification of Dyssynchrony
}

\author{
By
}

Michel Lalonde, B.Sc.

\begin{abstract}
A thesis submitted to
in partial fulfilment of

the degree requirements of

Master of Science

Ottawa-Carleton Institute for

Physics

Department of Physics

Carleton University

Ottawa, Ontario, Canada
\end{abstract}

The Faculty of Graduate Studies and Research

August 2008

Copyright $(\mathrm{C}$

2008 - Michel Lalonde 


$\begin{array}{ll}\begin{array}{l}\text { Library and } \\ \text { Archives Canada }\end{array} & \begin{array}{l}\text { Bibliothèque et } \\ \text { Archives Canada }\end{array} \\ \begin{array}{l}\text { Published Heritage } \\ \text { Branch }\end{array} & \begin{array}{l}\text { Direction du } \\ \text { Patrimoine de l'édition }\end{array} \\ \begin{array}{l}\text { 395 Wellington Street } \\ \text { Ottawa ON K1A 0N4 } \\ \text { Canada }\end{array} & \begin{array}{l}\text { 395, rue Wellington } \\ \text { Ottawa ON K1A 0N4 } \\ \text { Canada }\end{array}\end{array}$

Your file Votre référence ISBN: 978-0-494-44127-5 Our file Notre référence ISBN: 978-0-494-44127-5

NOTICE:

The author has granted a nonexclusive license allowing Library and Archives Canada to reproduce, publish, archive, preserve, conserve, communicate to the public by telecommunication or on the Internet, loan, distribute and sell theses worldwide, for commercial or noncommercial purposes, in microform, paper, electronic and/or any other formats.

The author retains copyright ownership and moral rights in this thesis. Neither the thesis nor substantial extracts from it may be printed or otherwise reproduced without the author's permission.
AVIS:

L'auteur a accordé une licence non exclusive permettant à la Bibliothèque et Archives Canada de reproduire, publier, archiver, sauvegarder, conserver, transmettre au public par télécommunication ou par l'Internet, prêter, distribuer et vendre des thèses partout dans le monde, à des fins commerciales ou autres, sur support microforme, papier, électronique et/ou autres formats.

L'auteur conserve la propriété du droit d'auteur et des droits moraux qui protège cette thèse. $\mathrm{Ni}$ la thèse ni des extraits substantiels de celle-ci ne doivent être imprimés ou autrement reproduits sans son autorisation.
In compliance with the Canadian Privacy Act some supporting forms may have been removed from this thesis.

While these forms may be included in the document page count, their removal does not represent any loss of content from the thesis.
Conformément à la loi canadienne sur la protection de la vie privée, quelques formulaires secondaires ont été enlevés de cette thèse.

Bien que ces formulaires aient inclus dans la pagination, il n'y aura aucun contenu manquant.

\section{Canada}




\section{Abstract}

Phase analysis of SPECT radionuclide angiography has been proposed, but not yet fully developed or validated, as a means of detecting cardiac dyssynchrony and predicting response to cardiac resynchronization therapy.

Regional, semi-automated SPECT radionuclide angiography phase analysis algorithms, including three methods of left ventricle motion sampling, were developed and validated. The ability of the algorithms to detect dyssynchrony and predict cardiac resynchronization therapy response was assessed using receiver operating characteristic (ROC) analysis. Phase parameters were able to detect dyssynchrony with ROC areas > 0.98 and predict cardiac resynchronization therapy response with $\mathrm{ROC}$ areas $<0.75$. These values are consistent with other published work utilizing different imaging techniques. Due to the semi-automated nature of the analysis, excellent correlation coefficients for intra- and inter-observer repeatability were reported.

Improved edge detection and use of the regional information to assess the pacing site would further enhance the clinical potential of SPECT radionuclide angiography phase analysis. 


\section{Acknowledgements}

I would like to thank my supervisors, Dr. Rob deKemp and Dr. Richard Wassenaar

for their guidance. In particular, I would like to thank Dr. Wassenaar for his patience and help.

I would like to thank my family, in particular my girlfriend, Jacynthe, for their constant support throughout my research.

Finally, thanks to everyone at the Ottawa Heart Institute and the Ottawa Hospital who have helped me during my research. I look forward to continuing my research there. 


\section{Table of Contents}

Abstract

Acknowledgements iii

Table of Contents iv

List of Tables $\quad$ vii

List of Figures $\quad$ viii

Acronyms

Overview $\quad$ xii

$\begin{array}{ll}\text { Chapter 1: Introduction } & 1\end{array}$

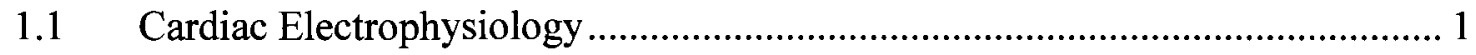

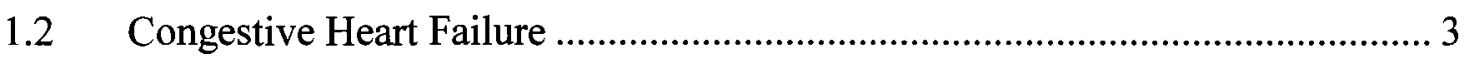

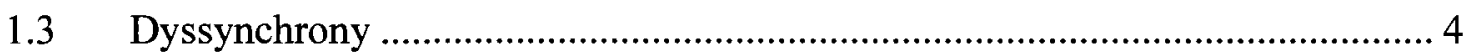

1.3.1 Types of Dyssynchrony: Electrical and Mechanical ............................... 5

1.3.2 Left Bundle Branch Block ..................................................................... 5

1.4 Cardiac Resynchronization Therapy .......................................................... 6

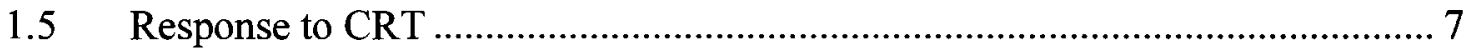

1.5.1 Electrical vs. Mechanical Dyssynchrony …........................................... 8

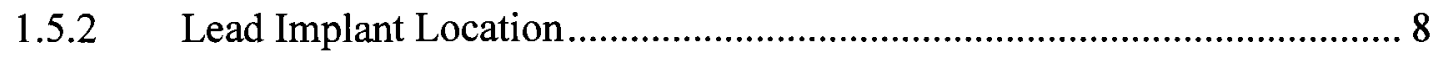

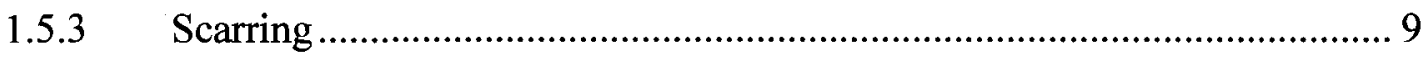

1.6 The Need to Quantify Mechanical Dyssynchrony ......................................... 9 


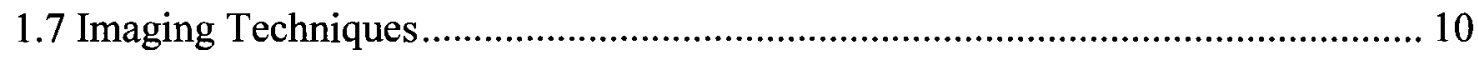

1.7.1 Echocardiography …............................................................................... 10

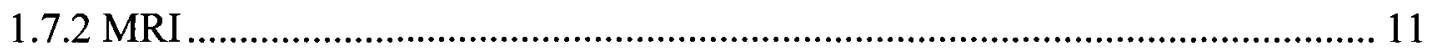

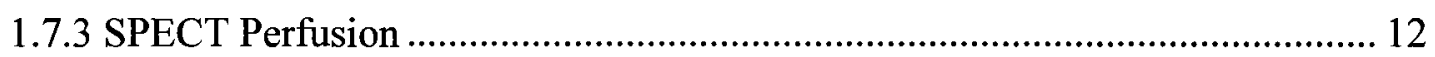

1.8 Radionuclide Angiography ......................................................................... 13

Chapter 2: Radionuclide Angiography $\quad 15$

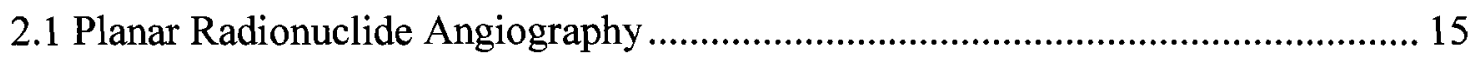

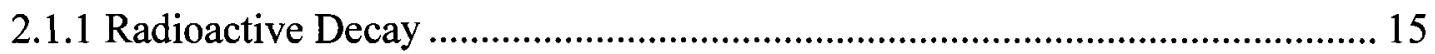

2.1.2 Radiotracer Labelling................................................................................. 16

2.1.3 Anger Camera …….................................................................................... 17

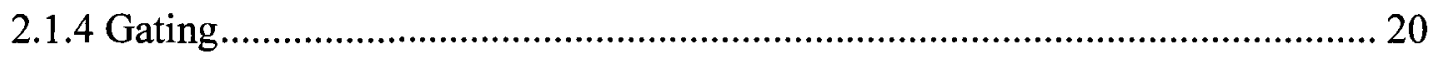

2.2 Phase Analysis of Planar RNA ………………................................................... 21

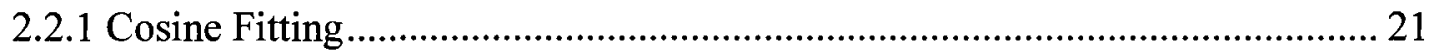

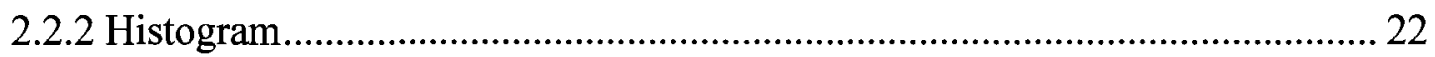

2.2.3 Quantification of Mechanical Dyssynchrony .................................................. 23

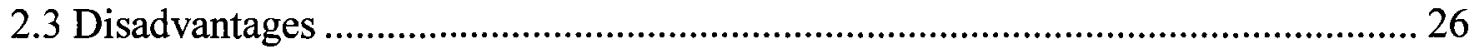

2.4 Single Photon Emission Computed Tomography RNA ......................................... 27

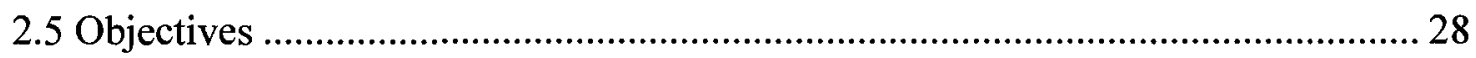

$\begin{array}{ll}\text { Chapter 3: Methods } & 30\end{array}$

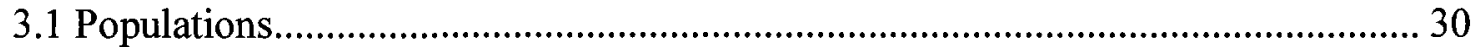

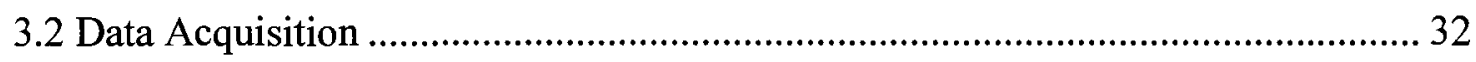

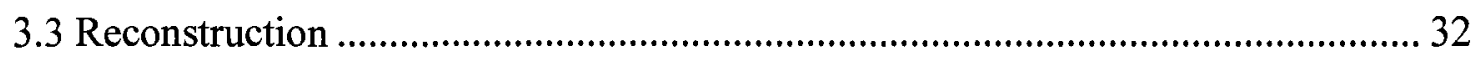

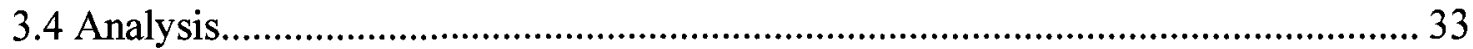

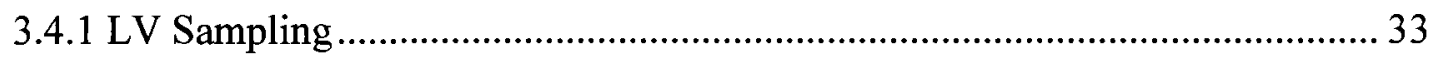

3.4.2 Wall Motion Analysis ................................................................................ 36

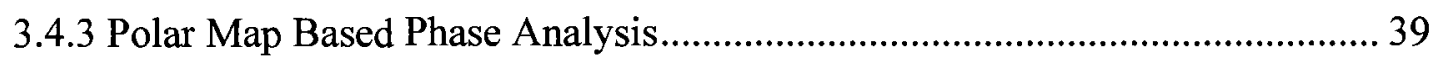

3.5 Validation Study ................................................................................................. 41

3.5.1 Validation of the Quantification of Mechanical Dyssynchrony ....................... 41 
3.5.2 Variability .....

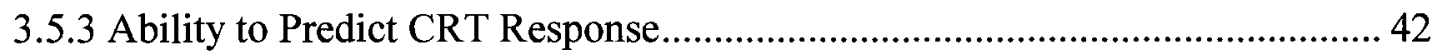

Chapter 4: Results $\quad 43$

4.1 Normal vs. Mechanical Dyssynchrony Population..................................................... 43

4.2 Observer Variability ....................................................................................... 47

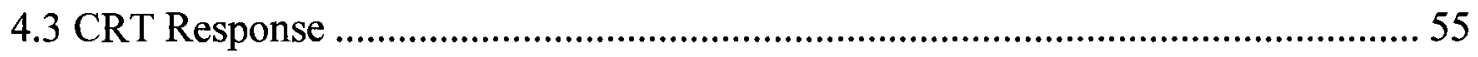

$\begin{array}{lr}\text { Chapter 5: Discussion } & 59\end{array}$

$\begin{array}{ll}\text { Chapter 6: Future Directions } & 67\end{array}$

$\begin{array}{ll}\text { Chapter 7: Conclusion } & 69\end{array}$

$\begin{array}{ll}\text { Appendix A: Software Development } & 70\end{array}$

Appendix B: Receiver Operator Characteristic Analysis $\quad 75$

$\begin{array}{ll}\text { References } & 77\end{array}$ 


\section{List of Tables}

Table 3.1: Patient demographics comparing populations................................................ 31

Table 3.2: Patient demographics comparing responders and non-responders.................. 31

Table 4.1: ROC areas for normal vs. mechanical dyssynchrony population..................... 43

Table 4.2: Intra- and inter-observer bias of Bland-Altman plots...................................... 55

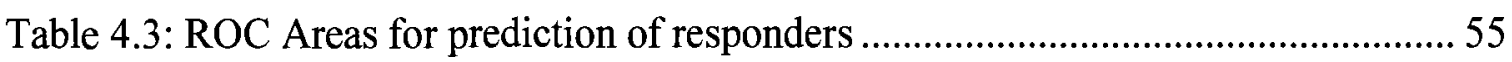

Table 5.1: Comparison of planar RNA and SPECT RNA ROC results ............................ 61

Table 5.2: Comparison of CRT response from various imaging modalitiese..................... 66 


\section{List of Figures}

Figure 1.1: Sample Electrocardiogram .................................................................... 3

Figure 1.2: Sample RNA image............................................................................. 14

Figure 2.1: Decay scheme of Technetium-99m ........................................................ 16

Figure 2.2: Diagram of an Anger camera .................................................................. 18

Figure 2.3: ECG of a heartbeat divided into 8 bins ...................................................... 21

Figure 2.4: Sample RNA image at various gates......................................................... 21

Figure 2.5: LV phase histograms of normal and mechanical dyssycnhrony patients...... 23

Figure 2.6: SPECT RNA slices with corresponding phase and amplitude images .......... 28

Figure 3.1: Schematic of conical/cylindrical sampling algorithm ................................... 34

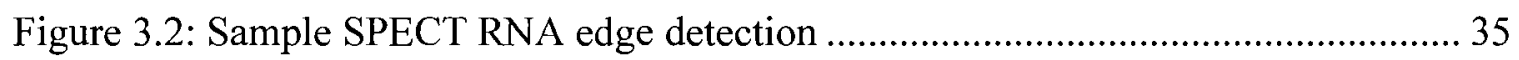

Figure 3.3: Time-activity based LV wall sampling ....................................................... 37

Figure 3.4: Time-distance based LV wall sampling ........................................................ 38

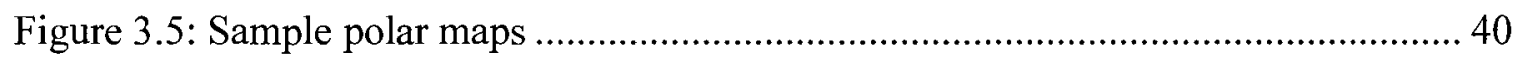

Figure 4.1: Phase SD ROC curves of normal vs. mechanical dyssynchrony patients...... 44

Figure 4.2: Synchrony ROC curves of normal vs. mechanical dyssynchrony patients.... 44

Figure 4.3: Entropy ROC curves for normal vs. mechanical dyssynchrony patients ....... 45

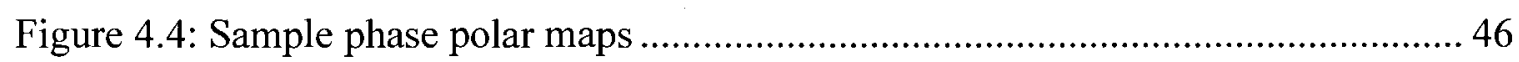

Figure 4.5: Time activity-based intra-observer regression and Bland-Altman plot ......... 48

Figure 4.6: Time distance float intra-observer regression and Bland-Altman plot ....... 49

Figure 4.7: Time distance fixed intra-observer regression and Bland-Altman plot ...... 50

Figure 4.8: Time activity-based inter-observer regression and Bland-Altman plot ......... 51

Figure 4.9: Time distance float inter-observer regression and Bland-Altman plot ......... 52

Figure 4.10: Time distance fixed inter-observer regression and Bland-Altman plot. ...... 53

Figure 4.11: ROC curves for phase SD for prediction of CRT responders ...................... 56 
Figure 4.12: ROC curves for synchrony for prediction of CRT responders...................... 56

Figure 4.13: ROC curves for entropy for prediction of CRT responders ......................... 57

Figure 4.14: Box-plot comparison of responders and non-responders............................. 58 


\section{Acronyms}

$\mathrm{CHF}$ Congestive Heart Failure

CHI. Contraction Homogeneity Index

CRT Cardiac Resynchronization Therapy

CV Coefficient of Variation

ECG Electrocardiogram

EF Ejection Fraction

HLA Horizontal Long Axis

LBBB Left Bundle Branch Block

LV Left Ventricle MPI Myocardial Perfusion Imaging MRI Magnetic Resonance Imaging NYHA New York Heart Association

PMT Photomultiplier Tube

ROC Receiver Operating Characteristic

ROI Region of Interest RNA Radionuclide Angiography RV Right Ventricle SA Short Axis 
SD Standard Deviation

SPECT Single Photon Emission Computed Tomography SPWMD Septal-Posterior Wall Motion Delay

VLA Vertical Long Axis 


\section{Overview}

Cardiac dyssynchrony occurs when the ventricles of the heart do not beat in phase. This can often reduce the pumping efficiency of the heart, leading to major cardiac problems, such as congestive heart failure. Cardiac resynchronization therapy (CRT) is a procedure in which a sophisticated pacemaker is surgically implanted and connected to the patient's heart, helping the regions of the ventricles contract on time relative to the rest of the ventricles, thus reducing dyssynchrony. However, CRT possesses a high rate of nonresponse, up to $50 \%(1)$. The reason for this high non-response rate is uncertain. Various medical imaging modalities have attempted to predict the outcome of CRT in order to better understand why certain patients do not respond to the CRT, and to help increase CRT response by better patient selection. Single photon emission computed tomography (SPECT) radionuclide angiography (RNA) has shown promise at predicting CRT response; however, methods for analysing the prediction of CRT response have not yet been fully developed. In this thesis, SPECT RNA phase analysis methods will be developed and validated to determine if it can properly predict CRT response.

In this thesis, a review of cardiac electrophysiology is presented in the Introduction chapter, followed by a discussion on various types of dyssynchrony. Chapter 1 continues with information on cardiac resynchronization therapy and the problem of non-response to this therapy. A literature review of existing medical imaging techniques previously 
used for assessment of cardiac resynchronization therapy response is given. Chapter 2 presents the imaging modality used for this thesis, SPECT RNA. Starting from the basic physics behind planar RNA and continuing with the description of the phase analysis methods required, SPECT radionuclide angiography is described in detail. Chapter 3 then presents the methods developed which were applied to validate the SPECT RNA techniques. This includes three left ventricle (LV) wall edge detection algorithms and polar map-based phase analysis. A validation study is also presented in this chapter. Chapter 4 presents the results of the validation study using receiver-operator characteristic analysis as well as correlation coefficients for intra- and inter-observer variability. The results and implications of this study are then discussed in chapter 5 . Chapter 6 then discusses future directions, followed by the conclusion in chapter 7. The software developed during this study to enable SPECT RNA analysis is presented in Appendix A. A detailed explanation of receiver operating characteristic analysis, which is used in this thesis, is presented in Appendix B 


\section{Chapter 1: Introduction}

\subsection{Cardiac Electrophysiology}

The heart is a vital organ that pumps blood, enabling oxygen and nutrients to reach the body. The heart is divided into 4 main chambers: the left atrium, the right atrium, the left ventricle (LV) and the right ventricle (RV). Pumping occurs when the heart contracts due to an electric stimulus. This electrical stimulus begins in the sinoatrial node, located in the right atrial wall, which acts as an internal pacemaker, initiating cardiac electrical activity, which propagates via an action potential. The action potential moves through the atrial fibres, initiating contraction of both atria. The right atrium contains oxygenpoor blood which is directed to the right ventricle, while the contraction of the left atrium sends oxygen-rich blood into the left ventricle. Following the atrial contraction, the action potential reaches the atrioventricular node located between the four main chambers of the heart, which then initiates an action potential in the left and right bundle branches that propagates the electrical signal to the LV and RV from the septal side of the ventricle, down to the apex and finally reaching the lateral side. The electrical signal, as it travels through both bundle branches then branches into the Purkinje fibres, which relays the action potential to the ventricular myocardium, causing contraction. During 
ventricular contraction, the right ventricle sends blood to the lungs for oxygenation, whereas the left ventricle ejects the oxygenated blood to arteries for the blood to reach all parts of the body. The left and right ventricles typically start to contract within $40 \mathrm{~ms}$ of each other when the action potential propagates through the specialized fibres discussed previously.

The electrical activation of the heart can be measured using an electrocardiogram (ECG), which measures electrical activity of the heart vs. time, as shown in Figure 1.1, using surface electrodes that record myocardial membrane polarization. The electrical activation of the atria is observed as the P-Q interval, whereas the Q-T interval represents the electrical activation of the ventricles as the action potential passes through both right and left bundle branches. The QRS interval, which marks the onset of ventricular contraction due to depolarization, is an important marker that is often used for the diagnosis of electrical dyssynchrony as described in section 1.3. The T wave corresponds to repolarization of the ventricles, which is longer in duration that the depolarization phase. 


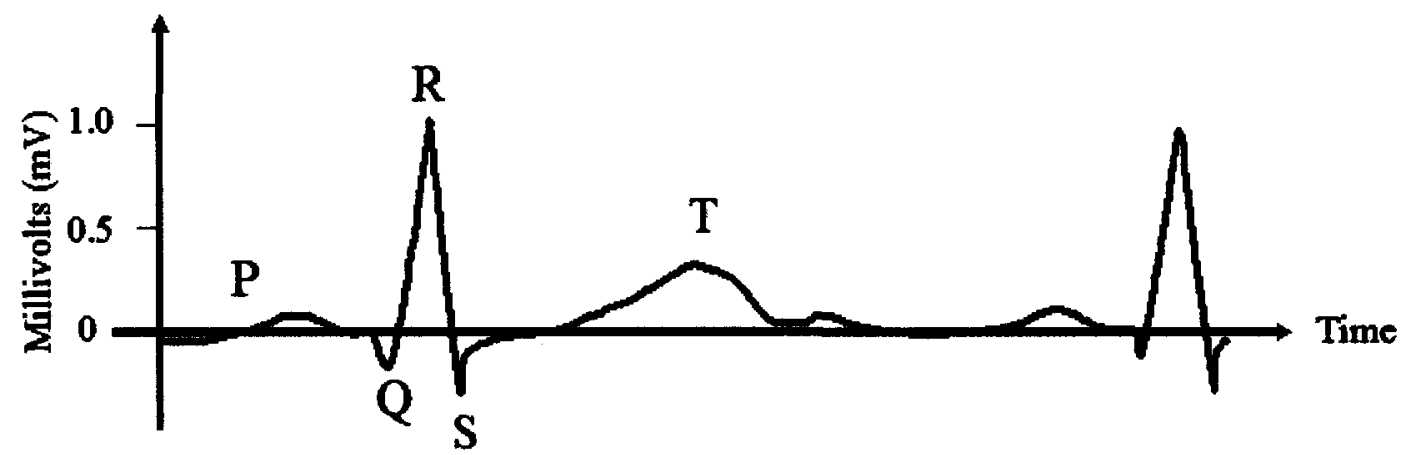

Figure 1.1: Sample Electrocardiogram, which plots electrical activity of the heart $(\mathrm{mV})$ as a function of time. Some important stages of a heartbeat are marked P, Q, R, S, and T. Of particular importance for this thesis, the QRS interval represents the onset of ventricular contraction

\subsection{Congestive Heart Failure}

Cardiovascular disease is the leading cause of death among men and women in Canada, totalling 36\% of all Canadian deaths in 1997 (2). Congestive heart failure (CHF) is one of the leading types of cardiovascular disease, behind only ischemic heart disease and cerebrovascular disease in both mortality (3) and hospital separations (4), which represents the number of patients leaving the hospital for any possible reason. CHF is a generic term that describes any disease in which the heart does not pump as strongly as it should. In order to compensate, the LV and RV can become enlarged thus increasing blood volume, ensuring enough blood flows through the body. The end-diastolic volume represents the amount of blood present in the LV when it is at its most dilated, just prior to the QRS interval. The end-systolic volume represents the blood volume of the LV when the LV is contracted at its maximum. The difference in these two volumes divided 
by the end-diastolic volume gives the ejection fraction $(\mathrm{EF})$ of the heart. An EF value under $35 \%$ is often used as a criteria for cardiac resynchronization therapy (CRT) selection (5-7).

The average length of stay for CHF based hospitalizations was just over 11 days in 2001/2002 (8). Adding to this burden was an elevated one-year re-admission rate for heart failure patients of 23.6 per 100 cases (9). It has been shown that cardiac mechanical dyssynchrony, described in section 1.3.1 is more prevalent with increasing severity of CHF (10).

\subsection{Dyssynchrony}

The ventricular walls of a healthy heart beat in phase and are said to be synchronous. The sub-optimal pumping efficiency stemming from CHF can be exacerbated by mechanical dyssynchrony. Mechanical dyssynchrony occurs when different regions of the heart wall beat out of phase with respect to each other, and can occur either inter- or intra-ventricularly. Inter-ventricular dyssynchrony implies that the left and right ventricles are not contracting in phase with respect to each other. Intra-ventricular dyssynchrony implies that within a single ventricle, different regions of the ventricle's wall contracts at different times. Both inter- and intra-ventricular dyssynchrony can lead to reduced pumping efficiency and low ejection fraction. 


\subsubsection{Types of Dyssynchrony: Electrical and Mechanical}

Electrical dyssynchrony refers to a delay in the electrical propagation of the action potential through the specialized fibers, which initiates cardiac contraction. The QRS duration obtained from an ECG is often used as a measure of electrical dyssynchrony. An abnormally prolonged QRS duration, usually quoted as greater than $120 \mathrm{~ms}$, can signal the presence of various types of electrical dyssynchrony, such as intra-ventricular conduction delay (11). It has been previously reported that electrical dyssynchrony is an independent risk factor of CHF $(10,12,13)$.

Mechanical dyssynchrony can be defined as a delayed timing of the physical contraction of some of the ventricular walls. It is the delay in the physical contraction of parts of the heart that leads to reduced pumping efficiency. In theory, the relationship between electrical dyssynchrony and mechanical dyssynchrony is highly variable between patients $(1,14-17)$. There are currently no well-established techniques that

provide a good assessment of mechanical dyssynchrony, despite the fact that it is mechanical dyssynchrony that may be of the most importance in assessing cardiac dyssynchrony (1).

\subsubsection{Left Bundle Branch Block}

Left bundle branch block (LBBB) is a type of electrical dyssynchrony in which there is a blockage of the action potential in the left bundle branch of the heart. Due to the 
blockage, the impulse from the action potential must propagate from cell to cell through the myocardium instead of following its normal course through the specialized fibres (11). This causes a delay of the propagating action potential from reaching the lateral wall or apex of the left ventricle through the electrical conduction system, thus resulting in a delay of the physical contraction of the LV lateral wall, with respect to the RV, since propagation is considerably slower (II). The lateral wall of a LBBB patient is expected to mechanically contract later due to the blockage of the action potential. However, this is not necessarily the case since mechanical and electrical dyssynchrony are not directly related. A delayed lateral wall contraction can be easily observed in a patient's phase polar map (discussed in section 3.4). In an LBBB patient, the QRS interval is often widened (>120 ms) due to this delay (11). Right bundle branch block is defined similarly for the right bundle branch.

\subsection{Cardiac Resynchronization Therapy}

Cardiac Resynchronization Therapy (CRT) is a relatively new surgical procedure that was approved by the Food and Drug Administration in 2001 (18). CRT consists of implanting a special pacemaker device in the patient. The pacemaker consists of three leads that are surgically connected to the patient's heart. The right atrial lead senses the natural onset of contraction originating from the sinoatrial node, and relays the information to the pacemaker. The pacemaker, pre-programmed with a certain time delay, then sends a signal to both the left and right ventricular leads, which initiates 
contraction in those regions. This type of pacing enables the section of the heart that receives an abnormal conduction delay to begin contracting immediately, thus attempting to improve cardiac synchrony. CRT has been shown to improve quality of life and decrease mortality and subsequent hospitalization rates $(1,5,15)$.

\subsection{Response to CRT}

There are, however, a large percentage of patients undergoing CRT who do not respond to the therapy. Non- responder percentages ranging from $30-50 \%$ can be found in the literature $(1,10,19)$.

There are currently no definitive criteria used to determine response to CRT. Hard clinical criteria such as patient death or heart transplant post-CRT, soft clinical criteria such as subsequent hospitalization for CHF, or improvements in New York Heart Association (NYHA) scores following therapy have been used to define response (1). NYHA scores range from class I (no limitations of activities) to class IV (patients should be at complete rest) (20). As well, one can use cardiac remodelling criteria to define response, such as a decrease in LV end-systolic volume by a certain amount, or improvement of LV ejection fraction by a certain amount. However, there is little consensus on a single criteria, and consequently responder results are dependent on the criteria used. Regardless of criteria selected, non-responder rates are elevated, and there may be several reasons for this high non-responder rate, as described in sections 1.5.1 through 1.5.3. 


\subsubsection{Electrical vs. Mechanical Dyssynchrony}

Currently, QRS duration, which is a measure of electrical dyssynchrony, is used to determine whether a patient is a good candidate for CRT (14). However, published results suggest that mechanical dyssynchrony and electrical dyssynchrony do not necessarily identify the same patients who qualify for CRT (1). Studies have shown that electrical dyssynchrony is not an ideal indicator of mechanical dyssynchrony $(6,14)$ and that proper assessment of mechanical dyssynchrony is key for patient selection and lead placement determination $(6,18)$. It has also been reported that improvement in electrical

dyssynchrony (i.e. altered QRS duration) was not a valuable predictor of response, again suggesting that electrical dyssynchrony and mechanical dyssynchrony are not well correlated (15).

\subsubsection{Lead Implant Location}

It has been shown that the location of ventricular lead implant can affect response to CRT (21). It has been also postulated that an optimal pacing location would be at the site of latest activation of the ventricle $(1,15)$. The site of latest activation is defined as the location on the ventricular wall for which the onset of mechanical contraction takes the longest to trigger. 


\subsubsection{Scarring}

The presence of scar tissue in the LV has been shown to reduce the responder rate to CRT (22). There are many causes of cardiac scarring, including ischemia and infarctions (23). However, it still remains unclear whether global cardiac scarring or localized scaring near the target LV pacing site decreases response. Scar tissue consists of fibrotic tissue, which is created during the tissue repair process when the cells responsible for restoration of function are unable to regenerate. Therefore myocardial fibrotic tissue is non-responsive to the incident electrical signal produced by the pacemaker or the heart. It may be beneficial to avoid placing any CRT leads near scar tissue to maximize chances of response.

\subsection{The Need to Quantify Mechanical Dyssynchrony}

An imaging technique capable of quantifying mechanical dyssynchrony and defining the site of latest activation may lead to improved patient care by properly screening candidates for CRT, as well as determining the ideal location of lead implants. This could potentially result in an increase in the number of responders to CRT and also increase the magnitude of response of current responders. In this thesis, single photon emission computed tomography (SPECT) radionuclide angiography (RNA) phase analysis (described in Chapter 2) is investigated as a means of imaging mechanical dyssynchrony. 


\subsection{Imaging Techniques}

Several other medical imaging techniques, described in this chapter, have been proposed as possibilities for accurately quantifying mechanical dyssynchrony. These include: echocardiography, magnetic resonance imaging (MRI), and SPECT perfusion imaging.

\subsubsection{Echocardiography}

Echocardiography has been investigated extensively for the assessment of mechanical dyssynchrony, notably due to its widespread availability and relatively low cost. Tissue Doppler imaging is the principal technique used amongst echocardiographic techniques for cardiac dyssynchrony analysis $(5,19)$. In order to quantify dyssynchrony, tissue Doppler imaging measures the velocity of various segments of the ventricular wall during a heart beat $(19,24)$.

While echocardiographic techniques have shown potential and encouraging results for identifying responders $(1,12,25,26)$ there are several challenges to this imaging technique. Sub-optimal quality echocardiographic images, which can occur in some patients (5), render interpretation difficult due to low resolution and the presence of artifacts. This can be due to misaligned images, due to incorrect axis acquisition, which can produce velocity curves consisting of both longitudinal motion and radial velocities, which are not desired (14). As well, echocardiographic analysis requires a fair amount of operator input, potentially increasing the inter-operator variability of the analysis.

A large (426 patients) international, multicenter study (PROSPECT (27)) investigated a variety of tissue Doppler imaging parameters to determine whether any of them could 
be used to predict response to CRT. Indicators of response to CRT used were an improvement of a committee reviewed clinical composite score, as well as a reduction of at least $15 \%$ in left ventricular end-systolic volume measured in a 6 month post-CRT follow-up scan. Intra-observer variability of tissue Doppler imaging parameters varied from $3.7 \%$ to $24.3 \%$. Inter-observer variability ranged from $6.5 \%$ to as high as $72.1 \%$ (7). The area under the receiver-operating characteristics curve for each predictor was no greater than 0.62 , indicating poor sensitivity and specificity results. The PROSPECT study concluded, based on poor reproducibility as well as poor ROC areas, that no single echocardiographic measure of dyssynchrony could be recommended for use in predicting CRT response (7).

\subsubsection{MRI}

Similar to tissue Doppler imaging in that it attempts to measure the velocity of the ventricular wall (28), velocity encoded MRI is sometimes used for mechanical dyssynchrony assessment. MRI possesses good temporal resolution, approximately twice that of SPECT techniques but three to four times less than that of tissue Doppler imaging (28). This technique has been compared to tissue Doppler imaging, and excellent correlation between the two techniques for a variety of parameters was reported (28). Another imaging technique called delayed enhancement MRI attempts to predict CRT response based on its detection of scar tissue using a technique called area planimetry. (29). The authors reported sensitivity and specificity values of $100 \%$ for the prediction of CRT response in a small population of 23 patients using percent of septal scar as 
response criteria. However, the nature of MRI technology prevents any patient implanted with a pacemaker to be imaged. This is due to strong magnetic fields present in MRI scans that would affect the metal components of the pacemaker, thus making follow-up scans challenging. Furthermore, it is an expensive technology that is currently not as readily available as other imaging modalities described in this thesis.

\subsubsection{SPECT Perfusion}

Also referred to as gated myocardial perfusion imaging (MPI), this nuclear medicine technique measures blood flow to the myocardial wall. SPECT MPI attempts to quantify mechanical dyssynchrony by measuring the change in the myocardial wall thickness as the heart beats. Wall thickness is related to wall motion since the density of cardiac muscle remains constant. Therefore, as the heart contracts, the wall must thicken. Due to partial volume effects, the measured activity (gamma-ray counts recorded per unit of time) of the cardiac wall changes as the wall thickens $(6,18,30,31)$. This change in measured activity can be plotted vs. time as a time-activity curve, which can then be used with phase analysis, similar to RNA phase analysis techniques described in Chapter 2, to determine regional wall contraction timing $(6,19,32)$. It is possible that regions of low perfusion, in which low activity is recorded, can result in faint or even missing sections of the reconstructed wall. This may cause difficulties in performing a complete phase analysis of the myocardial wall and is considered one of the main disadvantages of this technique. 
A recent SPECT perfusion study $(6,32)$ investigated how well it could predict response to CRT. An improvement of at least 1 level in the New York Heart Association (NYHA) functional class was used as a definition of response. An ROC area of 0.81 for the prediction of CRT using phase standard deviation (described in section 2.2.3), with sensitivity and specificity values of $74 \%$ were reported. Correlation coefficients of 0.99 or better for intra- and inter-observer repeat measurements indicated good intra- and inter- observer reproducibility of this technique (6).

\subsection{Radionuclide Angiography}

As described in greater detail in chapter 2, radionuclide angiography (RNA) is a nuclear medicine technique that images the chambers of the heart via a radioactive tracer tagged to red blood cells. A sample RNA image is shown in Figure 1.2. The total count recorded in each pixel of the image is proportional to the blood volume present in that pixel. Thus, it is possible to observe the beating cavities of the heart. Planar RNA is considered the gold standard for determination of $\mathrm{LV}$ ejection fraction $(33,34)$ and has previously been investigated as a tool to quantify mechanical dyssynchrony $(10,35)$. Phase analysis, as described further in section 2.2 , can be performed on planar RNA data to obtain a global measure of mechanical dyssynchrony. Previous works have investigated the capabilities of planar RNA at measuring mechanical dyssynchrony and predicting CRT response $(10,15,35)$, but SPECT RNA methods have not been fully developed. In this thesis work, SPECT RNA phase analysis methods were developed and 
validated in order to quantify mechanical dyssynchrony and determine whether it could predict response to CRT.

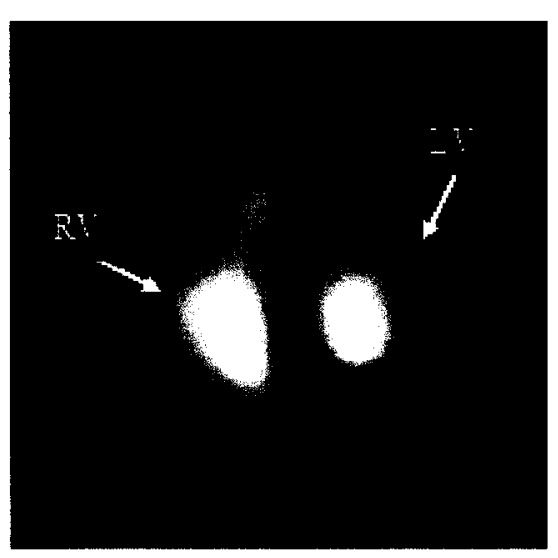

Figure 1.2: Sample RNA image at the end-diastolic frame. By tagging red blood cells with a radioactive tracer the ventricles of the heart can be imaged through the various phases of the contractile cycle. 


\section{Chapter 2: Radionuclide Angiography}

\subsection{Planar Radionuclide Angiography}

\subsubsection{Radioactive Decay}

An unstable isotope in a meta-excited state can stabilize by undergoing an isomeric transition, emitting a gamma-ray in the process. For RNA imaging, Technetium-99m (Tc-99m) is the radioisotope used. It possesses a half-life $\left(t_{1 / 2}\right)$ of 6.01 hours (36), which indicates that the activity of Tc-99m is reduced by half after 6.01 hours. This half-life provides sufficient time for the preparation of the radiotracer, while at the same time acquisition of the data can be accomplished in a reasonable time frame. Technetium- $99 \mathrm{~m}$ emits a $140.5 \mathrm{keV}$ gamma ray with $89 \%$ yield (36). This energy provides an acceptable trade-off between minimizing attenuation and patient dose while allowing for interaction with the detector (described in section 2.1.3). The decay scheme of Technetium-99m is shown in Figure 2.1. 


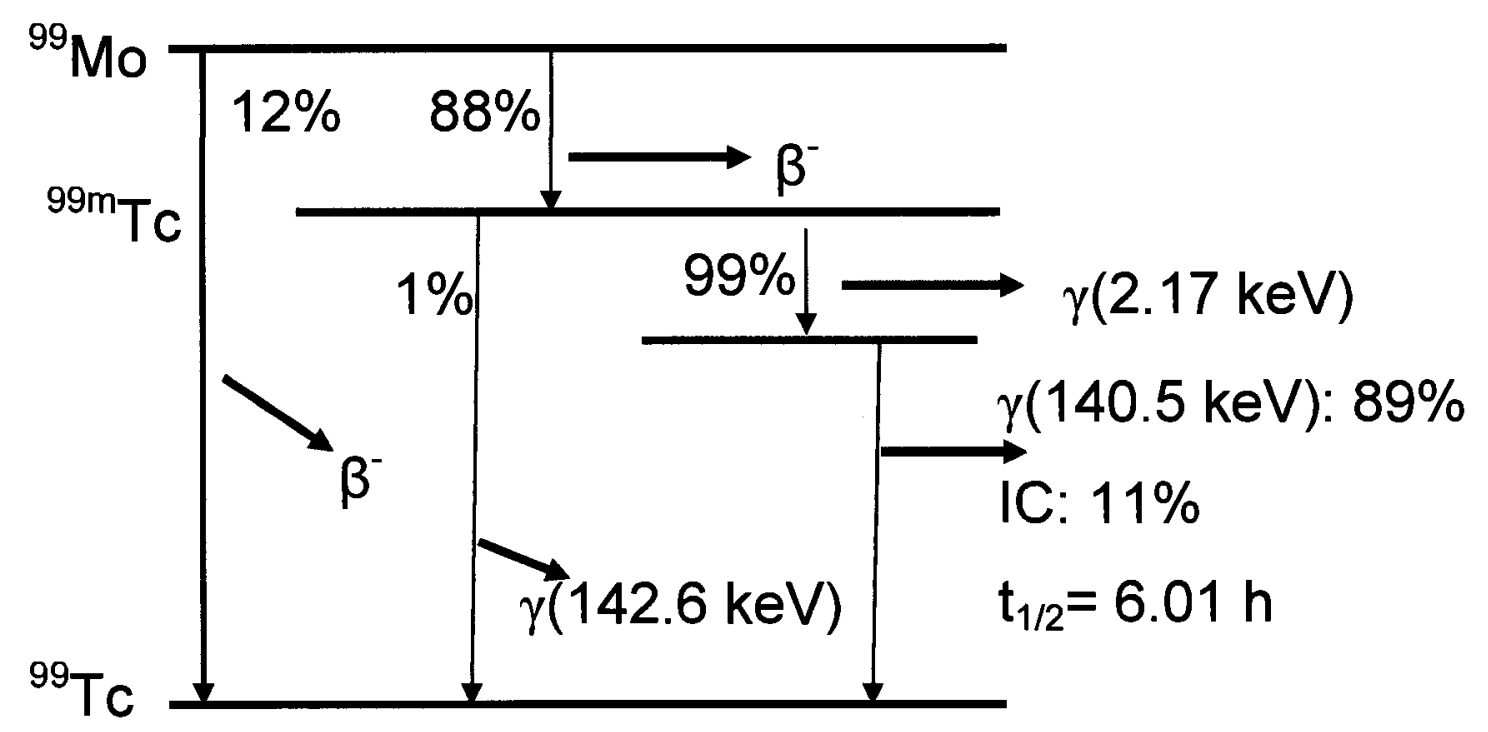

Figure 2.1: Decay scheme of Technetium-99m (not to scale). Important gamma-ray decays energies given in parentheses. IC represents internal conversion. The useful decay sequence starts at Molybdenum-99 $\left({ }^{99} \mathrm{Mo}\right)$ which decays via $\beta^{-}(89 \%)$ to ${ }^{99 \mathrm{~m}} \mathrm{Tc}$, which then decays via $\gamma$ to another excited state of ${ }^{99 \mathrm{~m}} \mathrm{Tc}(99 \%)$. By $\gamma$-ray $(140.5 \mathrm{keV}$, $89 \%$ ) emission or internal conversion (11\%), ${ }^{99 m} \mathrm{Tc}$ stabilizes to ${ }^{99} \mathrm{Tc}$, with a half-life of 6.02 hours. It is this $\gamma$-ray which is the used for SPECT imaging. (Adapted from: National Nuclear Data Center data. (36))

\subsubsection{Radiotracer Labelling}

The Technetium-99m radionuclide must be tagged onto a specific compound to become a radiotracer. Typically, this is a substance found in the human body, such as blood or sugar, which retains its properties after being labelled with a radionuclide. Once the radiotracer is injected into the patient, it will follow a certain physiological course, emitting gamma rays as it decays. It is then possible to locate uptake of the radiotracer inside the body with cameras designed to detect the gamma rays (section 2.1.3). 
In the case of RNA, the Technetium-99m is tagged directly to red blood cells. A blood sample is first taken from the patient. Technetium-99m is then mixed with the blood sample, tagging itself to the red blood cells. The tagged blood sample is then reinjected into the patient. Once the red blood cells tagged with Technetium-99m have dispersed evenly within the blood, it is possible to image areas of blood in the body, most notably the heart cavities.

\subsubsection{Anger Camera}

Once the patient has been injected with the Tc-99m tagged blood sample, an Anger camera is used to detect and localize the gamma rays which are emitted so that an image can be reconstructed. A diagram of the various components of an Anger camera is shown in Figure 2.2. This special type of camera consists of a lead collimator, a crystal scintillator, and a photomultiplier tube array.

The collimator, which consists of a slab of lead with many holes drilled in it, provides directional information regarding the gamma ray's origin. This is accomplished by preventing off-angle incident gamma rays. A parallel-hole collimator, which only allows gamma rays perpendicular to the surface of the detector through, is the most common type used. Other types of collimator include converging, diverging and pinhole collimators, which are used to increase or decrease the area being imaged (i.e. zoom). 


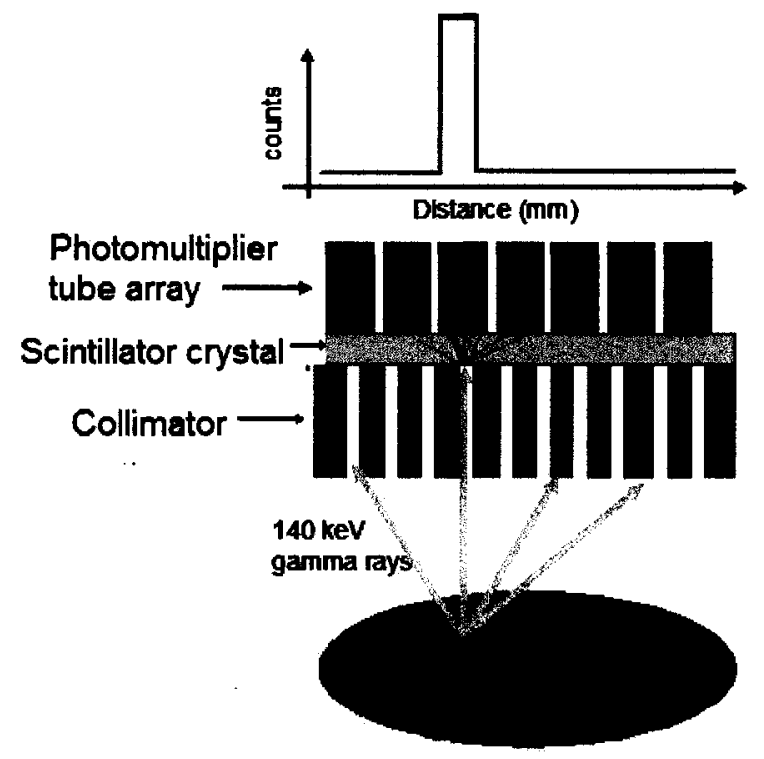

Figure 2.2: Diagram of the main components of an Anger camera. The collimator allows only gamma-rays perpendicular to the surface of the crystal to reach the crystal, thus providing directional information. The crystal and PMT assembly record and plot the location of the gamma-ray.

Scintillation detectors are widely-used as gamma-ray detectors with sodium iodide crystals doped with thallium $(\mathrm{NaI}(\mathrm{Tl}))$ being the crystal of choice for general nuclear medicine. The $140.5 \mathrm{keV}$ gamma-rays interact via the scintillation process with the detectors, emitting visible light photons in the process. More specifically, the incident gamma ray transfers its energy to an electron in the detector's crystal lattice. Many of these electrons are raised to excited states, some of which are caught in traps between energy levels created by the thallium impurities. Shortly after, the electrons return to the ground state, emitting photons in the process. Unlike the electrons caught in traps, electrons returning to the ground state from the conduction band will emit light photons with enough energy to re-excite other electrons, preventing the photon from escaping the 
scintillator and proceeding to PMT. The electrons from the energy traps will escape the scintillator and reach the photomultiplier tube array and produce photoelectrons. The relative light output of $\mathrm{NaI}(\mathrm{Tl})$ for a given gamma-ray interaction is excellent; it is the standard other crystals are compared to. It possesses a relatively high density of 3.7 $\mathrm{g} / \mathrm{cm}^{3}$, a decay time of $230 \mathrm{~ns}$ and emits photons in the $340-490 \mathrm{~nm}$ range (37).

The light emitted by the scintillator is then captured by a photon detector, most often a photomultiplier tube (PMT), where it is converted and amplified into an electrical signal. Light arriving at the PMT interacts with a photocathode via the photoelectric effect. The electrons emitted are then accelerated onto the first of a series of dynodes, each at a progressively higher voltage. This results in an amplification of the signal as more electrons are released from the interaction of the accelerated incident electrons with each dynode. The amplification process creates a signal large enough to be measured once it reaches the anode.

An Anger camera is comprised of an array of PMTs. A single scintillation event will produce a pulse height signal in many of the PMTs (38). It is then possible to sum up all these pulse signals and obtain a total signal, called the z-pulse, which is proportional to the energy of the incident gamma-ray (Eq. 2-1). An energy acceptance window is established to reject incident gamma-rays that have been scattered. It is also possible to determine the position of the incident gamma-ray on the detector by calculating the center of mass of the relative PMT signals using Eq. 2-2 and 2-3. 


$$
\begin{gathered}
Z=\sum_{k=1}^{K} a_{k}, \\
X=\frac{1}{Z} \sum_{k=1}^{K} x_{k} a_{k}, \\
Y=\frac{1}{Z} \sum_{k=1}^{K} y_{k} a_{k},
\end{gathered}
$$

where $\mathrm{Z}$ is the pulse height, $\mathrm{x}_{\mathrm{k}}$ and $\mathrm{y}_{\mathrm{k}}$ are the positions of the $\mathrm{k}^{\text {th }}$ PMT from a reference point, and $\mathrm{a}_{k}$ is the amplitude signal of the $\mathrm{k}^{\text {th }}$ PMT. An image is formed by creating a two-dimensional histogram of the gamma-rays, based on their position. For each recorded gamma-ray, an $\mathrm{X}$ and $\mathrm{Y}$ position is calculated and a value of 1 is added to the histogram bin, or pixel, corresponding to that $\mathrm{X}$ and $\mathrm{Y}$ coordinate. The counts in each pixel of the image represent the integral of the total activity in a volume defined by the pixel size, and extending in a direction defined by the collimator.

\subsubsection{Gating}

Using an electrocardiogram (ECG) signal, the cardiac cycle can be divided into a set number of bins, or gates (Figure 2.3). During RNA data acquisition, the counts are binned, not only in $\mathrm{X}$ and $\mathrm{Y}$ but also in the appropriate gate, based on the ECG trace. Data is acquired over many cardiac cycles to improve counting statistics, resulting in acquisition times of approximately 15 minutes. The result is an image at each gate, which can be combined to produce a movie of the beating heart (Figure 2.4). 


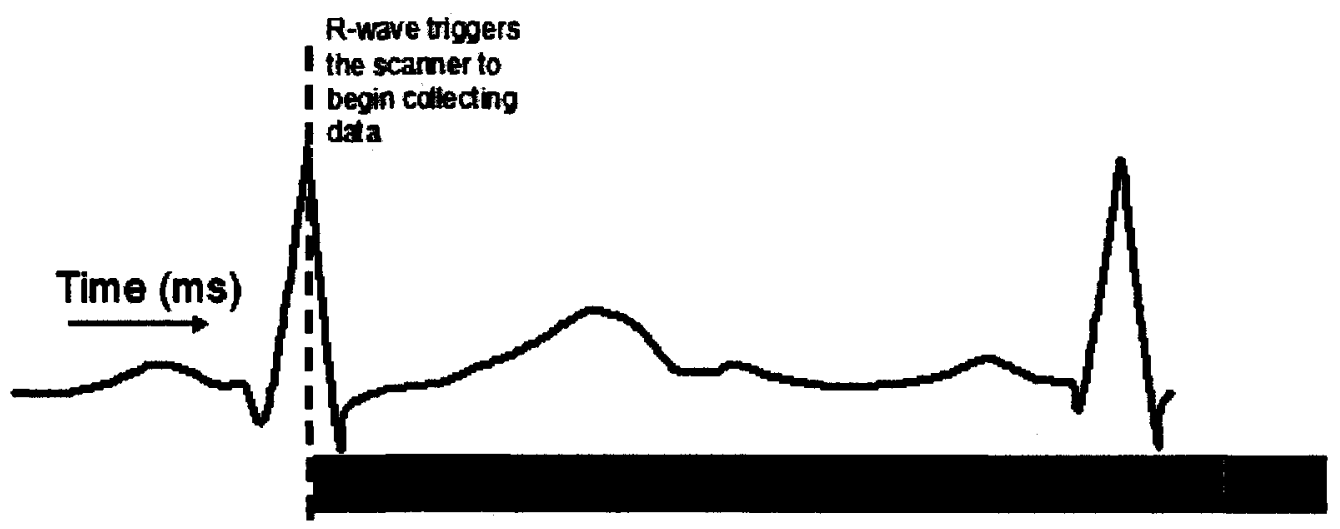

Figure 2.3: ECG of a heartbeat divided into 8 bins of equal time duration beginning at the Q-wave. The heartbeat can be divided into more bins, such as 16 or 24 .

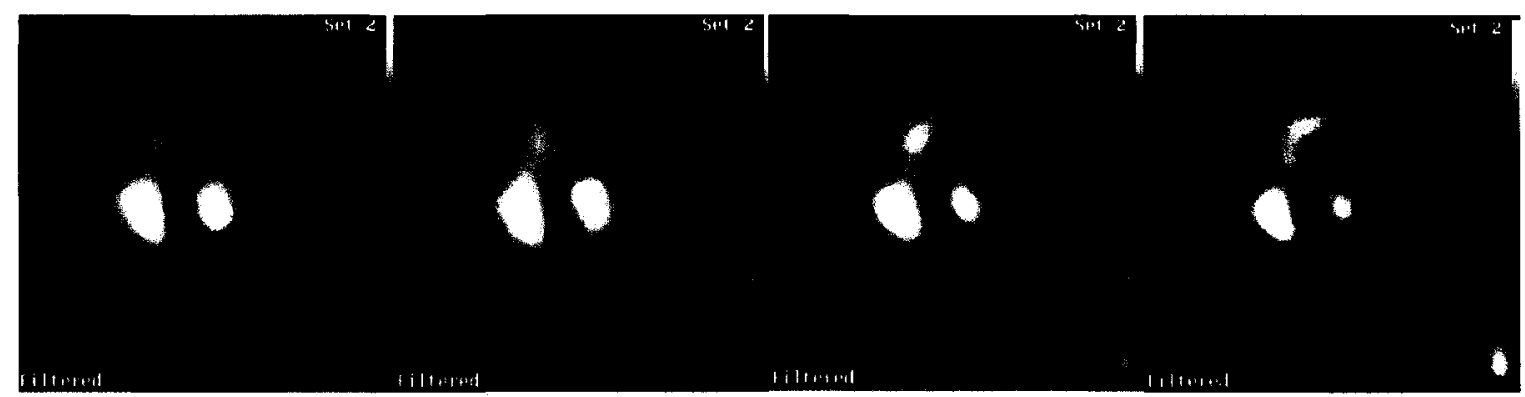

Figure 2.4: Sample RNA images of a short axis slice where the heartbeat was gated using 24 bins. Shown are gates 1, 3, 5 and 7 (out of 24).

\subsection{Phase Analysis of Planar RNA}

\subsubsection{Cosine Fitting}

Cardiac wall motion can be approximated as a sinusoidal wave. Fourier analysis can then be performed (Eqs. 2-4, 2-5 and 2-6) to extract a phase (Eq. 2-7) and amplitude (Eq. 2-8) value from the gated counts on a pixel by pixel basis (39). This is a discrete fit of the counts of a data point through all the gates in the cardiac cycle. 


$$
\begin{gathered}
F_{K}(f)=\sum_{i=1}^{N}\left(R_{K}-i I_{K}\right), \\
R_{K}(f)=\sum_{t=1}^{N} f(t) \cos (2 \pi K t / N), \\
I_{K}(f)=\sum_{t=1}^{N} f(t) \sin (2 \pi K t / N),
\end{gathered}
$$

where $R_{K}$ and $I_{K}$ represent the real and imaginary values, $f(t)$ is the value of pixel, $f$, at the $\mathrm{t}^{\text {th }}$ gate, $\mathrm{N}$ is the total number of gates, and $\mathrm{K}$ is the harmonic number.

$$
\begin{aligned}
& A_{K}(f)=\left(R_{K}(f)^{2}+I_{K}(f)^{2}\right)^{1 / 2}, \\
& P_{K}(f)=\arctan \left(I_{K}(f) / R_{K}(f)\right),
\end{aligned}
$$

where $A_{K}$ is the amplitude, and $P_{K}$ is the phase offset of the Fourier fit, for the $K^{\text {th }}$ harmonic. For the first harmonic, $\mathrm{K}=1$, amplitude, $\mathrm{A}_{1}$, corresponds to the extent of cardiac wall motion, and phase, $\mathrm{P}_{1}$, corresponds to the onset of contraction just after the end-diastolic time frame occurs. It may be possible to use $A_{1}$ and $P_{1}$ to quantify mechanical dyssynchrony.

\subsubsection{Histogram}

A phase histogram plotting the frequency of the LV phase angles can then be created. The histogram enables assessment of the distribution of the phases in the LV. In a healthy heart, a typical phase histogram contains a single, narrow peak, since the entire $\mathrm{LV}$ wall is beating in phase (Figure $2.5 \mathrm{a}$ ). In a heart with mechanical dyssynchrony, the 
phases are typically more spread out (Figure $2.5 b$ ). The histogram provides a qualitative assessment of mechanical dyssynchrony.

a)

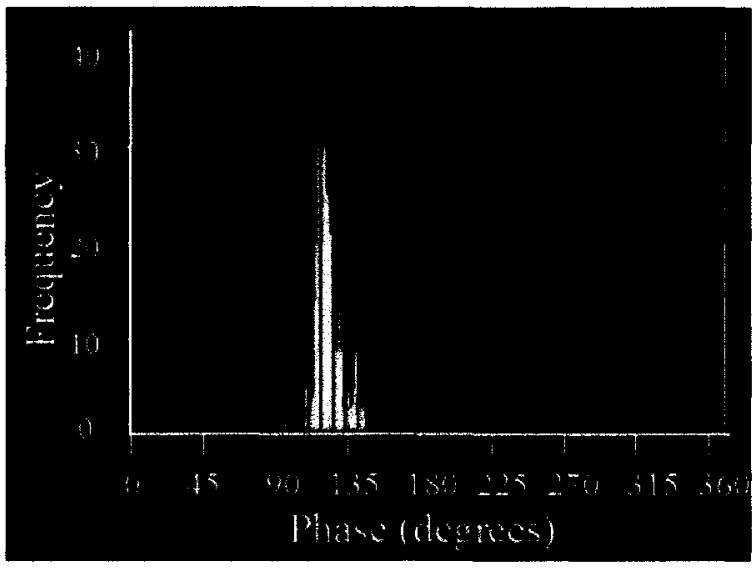

b)

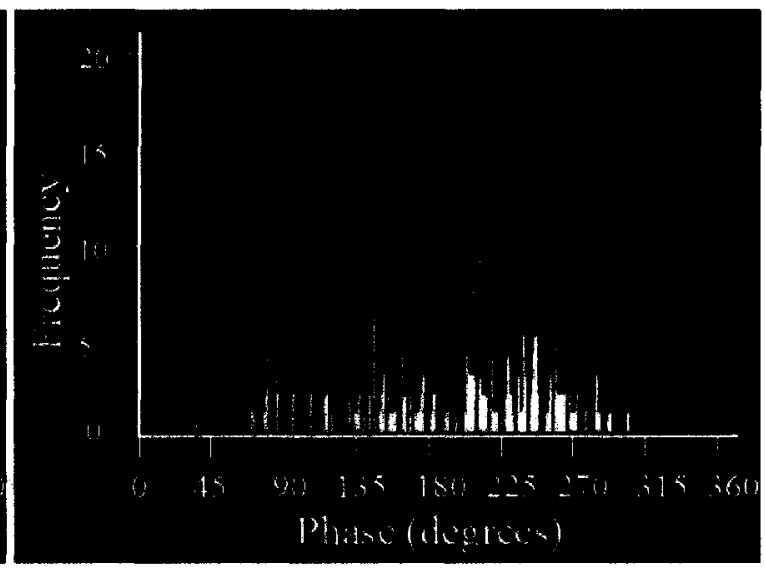

Figure 2.5: a) LV phase histogram of a patient with normal cardiac function. The distribution is single-peaked and narrow indicating high synchrony. b) LV phase histogram of a patient diagnosed with mechanical dyssynchrony. The phase distribution is broader than in a), indicating presence of dyssynchrony.

\subsubsection{Quantification of Mechanical Dyssynchrony}

Several parameters have been proposed to provide a single global value which represents a quantitative measure of mechanical dyssynchrony.

\section{Phase Standard Deviation}

The phase standard deviation (SD) of the histogram can be calculated, to provide a measure of the spread in phase values. A larger spread is expected to correspond to mechanical dyssynchrony (Figure 2.5b). Phase SD has been used in many previous 
phase analysis studies with mixed results $(10,15,18,19,35,40,41)$. O'Connell et al. (35) found that phase SD was able to differentiate normal cardiac synchrony from simulated forms of dyssynchrony ( $\mathrm{p}<0.05$ ), but was unable to differentiate between various types of dyssynchrony (aneurysm patients, severe diffuse dysfunction patients, and severe regional dysfunction patients).

\section{Synchrony}

First suggested by O'Connell et al. (35), synchrony, S, is a parameter which incorporates both phase and amplitude. It is defined in Eq. 2-9.

$$
S=\frac{\left|\sum_{i=1}^{N} \boldsymbol{v}_{\boldsymbol{i}}\right|}{\sum_{i=1}^{N}\left|\boldsymbol{A}_{\boldsymbol{i}}\right|},
$$

where $v_{i}$ represents a vector defined for pixel $i$, with a length given by amplitude $\mathrm{A}_{i}$ and a direction given by the phase. The numerator is the vector sum of all pixel vectors. The denominator, a normalization constant, is the sum of all the amplitude values. Synchrony values range from 0 (complete dyssynchrony) to 1 (complete synchrony). These two extreme cases are easily defined. If the vectors are oriented isotropically (i.e. complete dyssynchrony), their vector sum will cancel out, giving a synchrony score of 0 . However, if all the vectors are lined up (i.e. they all have the same phase value), synchrony will be 1 , indicating perfect synchrony. O'Connell et al. (35) performed a planar RNA phase analysis simulation study and found that synchrony values were 
significantly different $(\mathrm{p}<0.05)$ for all four groups compared. Synchrony has yet to be evaluated as a predictor of CRT response.

Recently, Harel et al. (42) suggested that a parameter called contraction homogeneity index (CHI) (Eq. 2-10), may be useful for the prediction of CRT response. The parameter's values range from -100 to 100 .

$$
C H I=\frac{\sum_{i=1}^{N}\left(A_{i} \cos \left(\theta_{i}\right)\right)}{\sum_{i=1}^{N} A_{i}} * 100 \%,
$$

where $\mathrm{N}$ is the total number of pixel vectors, $\mathrm{A}_{i}$ is the amplitude of the $i^{\text {th }}$ pixel vector, and $\theta_{i}$ is the angle of the pixel vector with respect to the average phase value of all pixel vectors. Similar to synchrony, CHI performs a normalized vector sum to quantify mechanical dyssynchrony. It was investigated in our lab, but was found to produce similar results to synchrony and was therefore not included in further analysis.

\section{Entropy}

Like synchrony, entropy ,E, was first suggested by O'Connell et al. (35). Entropy can be defined as a measure of the randomness of the phase distribution. It is derived from Shannon information theory and is given in Eq. 2-11:

$$
E=\frac{-\sum_{i=1}^{M} P_{i} \log _{2} P_{i}}{\log _{2} M},
$$


where $\mathrm{M}$ is the number of bins in the histogram and $\mathrm{P}_{i}$ is the probability of having a phase value in bin $i$. Entropy values range from 0 (complete order) to 1 (complete disorder). Entropy can be summarized as follows: based on the existing phase distribution, what is the probability of accurately determining what the next phase value would be. If every single phase value is located inside the same bin, then the probability that the next value will fall there is definite and entropy is 0 . If each bin possesses the same frequency count, then the next value could probabilistically be anywhere and the entropy score is 1. Entropy is similar to phase SD since it is a measure of the histogram shape. However, it may provide complementary information to phase SD.

As with the results obtained for synchrony, $\mathrm{O}^{\prime}$ Connell et al. (35) found that entropy values were significantly different $(p<0.05)$ between four groups with various forms of mechanical dyssynchrony. Entropy also has yet to be evaluated as a predictor of CRT response.

\subsection{Disadvantages}

There are some limitations to planar RNA phase analysis. Shadowing effects can occur due to overlapping structures of the ventricle. This prevents analysis of movement perpendicular to the detector (33) as well as the localized detection of any abnormalities (34). Also, due to the 2D nature of the technique, true regional phase information cannot be obtained for the entire LV myocardium. 


\subsection{Single Photon Emission Computed Tomography RNA}

Single photon emission computed tomography (SPECT) RNA is the three-dimensional equivalent of planar RNA. The principles behind it are similar except that it is capable of producing 3D images by reconstructing data acquired over many projections. A single projection is equivalent to a planar RNA image. Once sufficient counts have been recorded in a single projection, the Anger camera is rotated and the process is repeated for the next projection. Using reconstruction methods such as filtered back projection, it is then possible to reconstruct the projection data into a three-dimensional representation of the activity distribution. As with planar RNA, SPECT RNA is gated, thus providing a three-dimensional cine of the beating heart. Phase analysis (section 2.2) can then be performed on each pixel (Figure 2.6). SPECT RNA does not suffer from shadowing effects and has been shown to improve assessment of regional wall motion in comparison with planar RNA (33). However, due to the need to collect multiple projections at each gate, fewer gates are recorded to improve counting statistics, thus potentially reducing temporal resolution. 


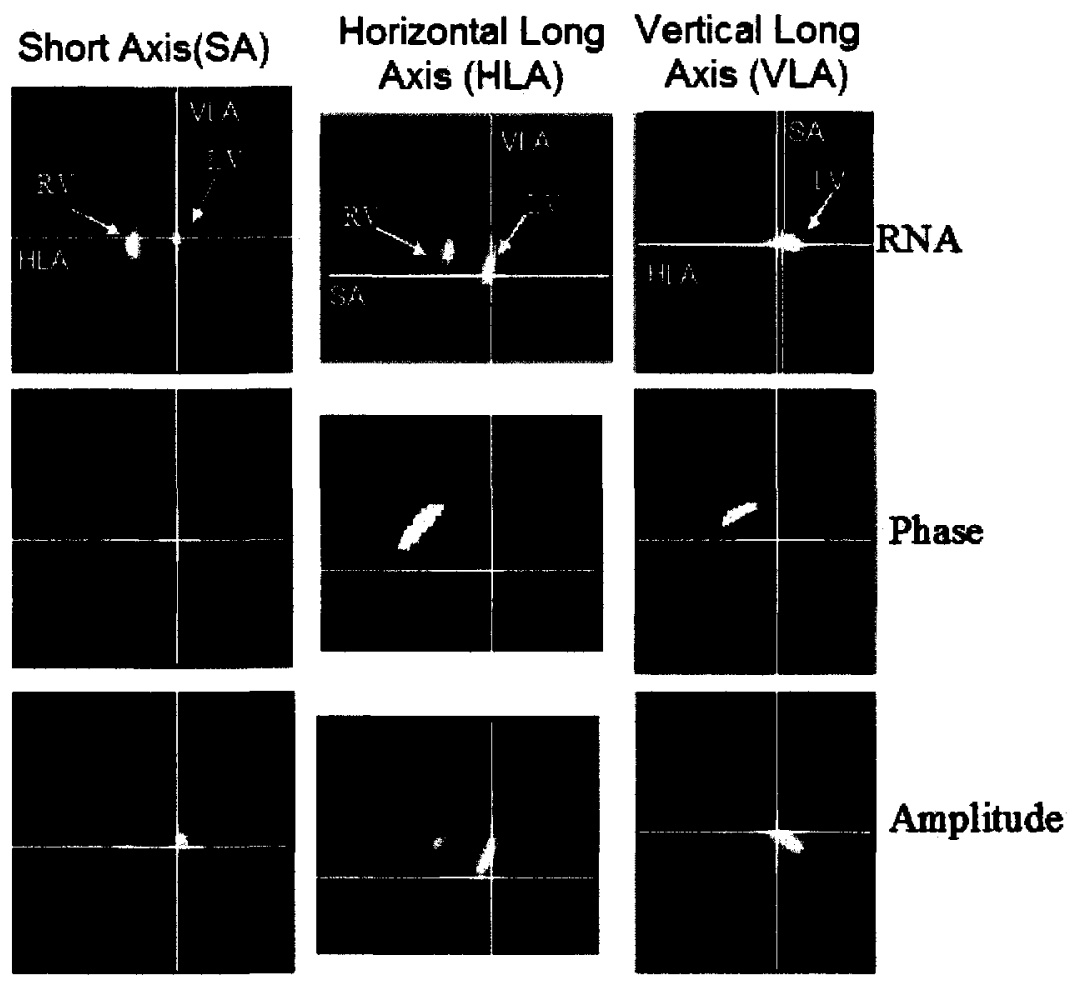

Figure 2.6: Top row: Various slices of the heart from a SPECT RNA study. Middle row: Corresponding phase images obtained by performing a Fourier transform and obtaining a phase value for each pixel in the image. Bottom row: Corresponding amplitude images obtained by performing a Fourier transform and obtaining an amplitude value for each pixel in the image.

\subsection{Objectives}

Previous SPECT RNA phase analysis studies have reconstructed a single, thick, planar slice from the SPECT data and have performed planar phase analysis (43),(44). There are currently no well established SPECT RNA methods capable of regionally assessing mechanical dyssynchrony using the full 3D data set. Methods of LV wall sampling for SPECT RNA have not been investigated. The potential of phase parameters synchrony 
and entropy to diagnose mechanical dyssynchrony have only been investigated using planar RNA (35). As well, no study has investigated their potential at predicting CRT response. Also, SPECT RNA phase parameter intra- and inter-observer variability has yet to be investigated.

The purpose of this research was to develop and validate SPECT RNA phase analysis algorithms. A polar map (defined in section 3.4.4) based, semi-automated LV wall sampling algorithm was developed. Regional phase values were determined using three methods of assessing wall motion, not previously investigated. Quantification of mechanical dyssynchrony was evaluated using three different parameters: phase SD, which is well-established, as well as synchrony and entropy, which have not yet been fully investigated. The algorithms developed were validated using ROC analysis on a population of normal subjects and a population with mechanical dyssynchrony. Intraand inter-observer variability were evaluated. Following this, a study assessing the ability of SPECT RNA in predicting response to CRT was undertaken. In summary, the objectives of this thesis were twofold: to develop a SPECT RNA phase analysis techniques capable of accurately quantifying mechanical dyssynchrony, and to determine whether mechanical dyssynchrony can be used to predict CRT response. 


\section{Chapter 3: Methods}

\subsection{Populations}

A population of 13 patients with normal electrical and mechanical cardiac function $(\mathrm{QRS}<120 \mathrm{~ms}$, LVEF $>55 \%$ ), and a group of 60 patients with known mechanical dyssynchrony were used to characterize and validate the methodologies developed in this thesis work. Signed consent was obtained for each subject included in this thesis. The patient demographics are presented in Table 3.1. The normal population were chemotherapy patients referred for baseline SPECT RNA for assessment of chemotherapy cardiotoxicity. The mechanical dyssynchrony population were patients with known mechanical dyssynchrony, as assessed by an expert observer, undergoing CRT. 58 of the 60 mechanical dyssynchrony patients were clinically diagnosed with LBBB.

A pre-CRT and 6 month follow-up post-CRT SPECT RNA scan were obtained for each patient in the mechanical dyssynchrony population. Using the 6 month follow-up scans of the mechanical dyssynchrony population, the criteria for a positive response to CRT was a $15 \%$ reduction in LV end-systolic volume, as assessed on the 6 month followup scan. This remodelling criteria is commonly used $(1,7,27)$. 11 of the 60 patients were excluded from the response to CRT analysis because their 6 month follow-up scan 
had not yet been performed. Of the 49 remaining patients, 25 (51\%) were classified responders. The demographics of these groups are shown in Table 3.2.

Table 3.1: Patient demographics comparing the normal and mechanical dyssynchrony populations.

\begin{tabular}{|c|c|c|}
\hline & Normal $(\mathrm{N}=13)$ & Mechanical dyssynchrony $(\mathrm{N}=60)$ \\
\hline Male/Female & $9 / 4$ & $50 / 9$ \\
\hline Age & $57 \pm 16 \mathrm{y}$ & $68 \pm 10 \mathrm{y}$ \\
\hline LV Ejection Fraction & $61 \pm 6 \%$ & $22 \pm 8 \% *$ \\
\hline End-systolic Volume & $42 \pm 9 \mathrm{~mL}$ & $230 \pm 98 \mathrm{~mL}^{*}$ \\
\hline End-diastolic volume & $107 \pm 17 \mathrm{~mL}$ & $291 \pm 99 \mathrm{~mL}^{*}$ \\
\hline QRS duration & $88 \pm 10 \mathrm{~ms}$ & $168 \pm 25 \mathrm{~ms}^{*}$ \\
\hline
\end{tabular}

${ }^{*} \mathrm{p}<0.05$ vs. normal

Table 3.2: Patient demographics of mechanical dyssynchrony population divided into responders and non-responders.

\begin{tabular}{|c|c|c|}
\hline & \multicolumn{2}{|c|}{ Mechanical dyssynchrony Population (N=49) } \\
\hline & Responder (N=25) & Non-Responder (N=24) \\
\hline Male/Female & $19 / 6$ & $22 / 2$ \\
\hline Age & $67 \pm 10 \mathrm{y}$ & $67 \pm 11 \mathrm{y}$ \\
\hline LV Ejection Fraction & $23 \pm 6 \%$ & $20 \pm 9 \%$ \\
\hline End-systolic Volume & $225 \pm 67 \mathrm{~mL}$ & $247 \pm 125 \mathrm{~mL}$ \\
\hline End-diastolic volume & $288 \pm 74 \mathrm{~mL}$ & $301 \pm 125 \mathrm{~mL}$ \\
\hline QRS duration & $173 \pm 22 \mathrm{~ms}$ & $163 \pm 27 \mathrm{~ms}$ \\
\hline
\end{tabular}

All demographics: $\mathrm{p}>\mathbf{0 . 0 5}$ for responder vs. non-responder 


\subsection{Data Acquisition}

Data was acquired using a dual-head gamma camera with low-energy high resolution collimators. The heads were positioned in a $90^{\circ}$ configuration. Projection data was acquired for $25 \mathrm{~s}$ per projection in a $64 \times 64$ matrix. 60 projections were acquired over $180^{\circ}$ in angular steps of $3^{\circ}$. A 3-lead ECG was used to gate each projection into 8 gates, based on a $10 \%$ beat-rejection window. This is the standard clinical protocol at the Ottawa Heart Institute, where the patients were imaged.

\subsection{Reconstruction}

Transverse slices were reconstructed into a 64 x 64 matrix using a filtered back projection algorithm, resulting in $6.88 \mathrm{~mm}$ pixel size. Data was smoothed using a $5^{\text {th }}$ order Butterworth filter with a cutoff of 0.35 of the Nyquist frequency.

The resulting images were resampled into 128 x 128 using a cubic spline interpolation algorithm. This was performed to increase the precision of the edge detection algorithm as pixel size became $3.44 \mathrm{~mm} \times 3.44 \mathrm{~mm}$. The transverse slices were then re-oriented into a set of short axis slices. 


\subsection{Analysis}

The following analysis was designed to be a semi-automated algorithm in order to reduce operator dependent results. For further details on the software development, see Appendix A.

\subsubsection{Sampling}

The LV wall was sampled using a cylindrical/conical sampling algorithm (Figure 3.1). The LV from apex to base was resampled into 18 slices. The locations of the base and apex were user-defined on the long-axis slices. The first 9 slices were apical and a conical sampling algorithm was used. The other 9 slices were basal slices and a cylindrical sampling algorithm was performed. For each slice, radial profiles were defined at various positions around the wall (Figure 3.1b). The very first apex edge contained a single LV sampling point, the next apical slice contained $9 \mathrm{LV}$ sampling points, the next slice had $18 \mathrm{LV}$ sampling points, and the remaining 15 slices contained $36 \mathrm{LV}$ sampling points each. This resulted in 568 radial profiles available for analysis of regional wall motion. 
a)

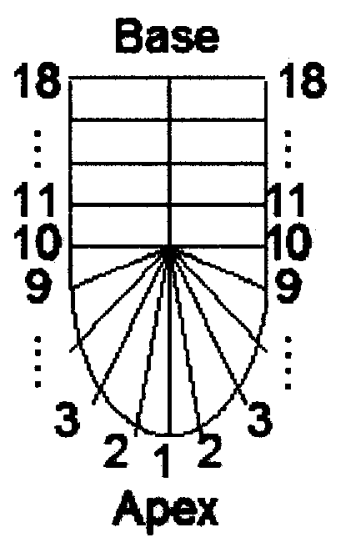

b)

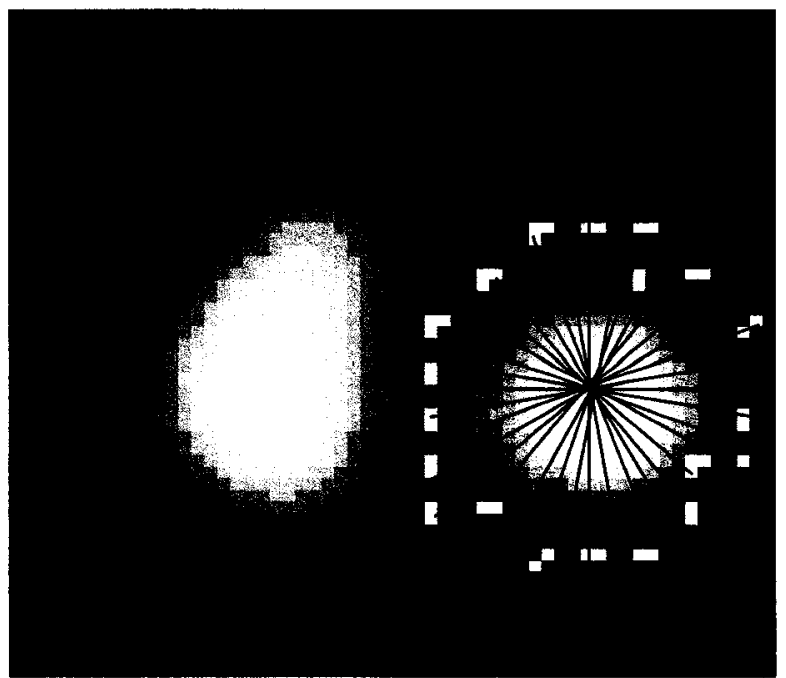

Figure 3.1: a) Schematic representation of canonical/cylindrical sampling algorithm. Slices 1-9 represent the apex while slices 10-18 represent the base. b) Short axis view of slice 11 (basal) with 36 radial projections.

For each radial projection defined in section 3.4.1, the LV edge was defined where the activity fell below $50 \%$ of the maximum activity found in the slice (Figure $3.2 \mathrm{~b}$ ). Linear interpolation was then performed using the pixels on either side of the $50 \%$ value to determine the location of the edge. Due to spill-over from the RV, the $50 \%$ threshold algorithm could not be used to determine the LV edge within the septum. Instead, the location of the minimum along the radial projection was taken as the edge. An example of a short axis slice is shown in Figure 3.2a. Figure 3.2b represents a corresponding counts profile across the short axis. 
a)

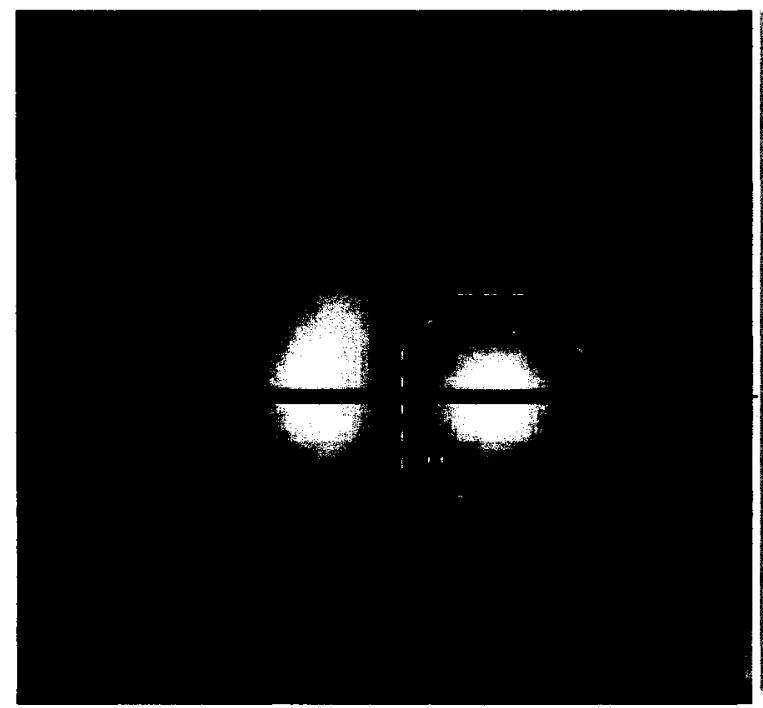

b)

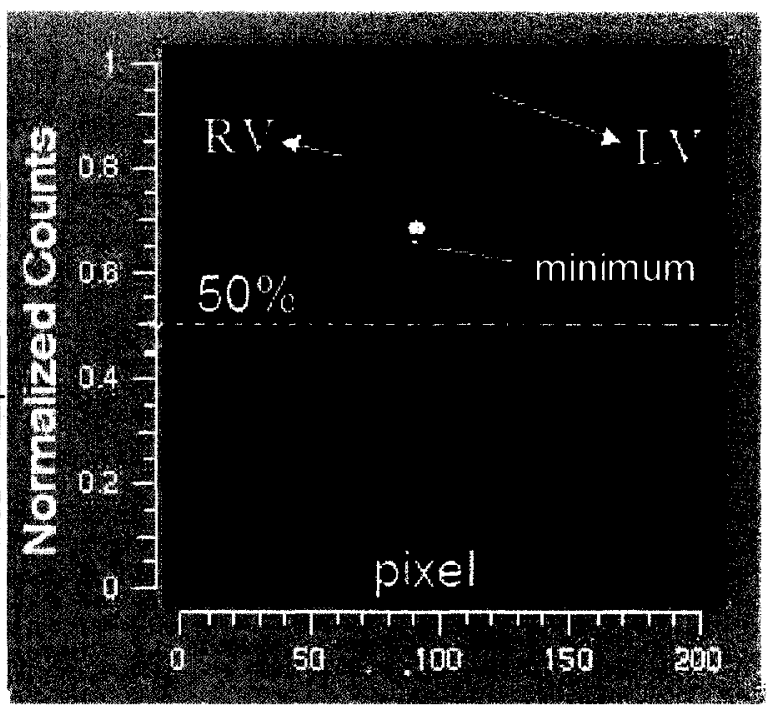

Figure 3.2: a) SPECT RNA short axis slice with the LV contour shown in green obtained using edge detection alogrithms, and b) normalized counts profile corresponding to the red line observed in a). The profile is normalized to the maximum count in the slice. Using the profile, the edge detection algorithm calculates two LV edge points, in this case, based on the $50 \%$ threshold (lateral side) and the minimum (septal side). These two $\mathrm{LV}$ edge points correspond to the intersection of the red line and green LV contour shown in a).

The threshold technique was chosen since it was intuitive, simple to implement, and provided robust edge detection. There exist other edge detection algorithms, such as the Sobel and Canny algorithms, that utilize a first order derivative of the image, enabling the algorithm to identify regions with the greatest rate of change, suggesting the presence of an edge. Both of these algorithms were initially investigated but not pursued due to poor initial results, possibly related to image noise. 
Invariant Laplacian edge detection, used by Harel et al. (33) for SPECT RNA calculated the Laplacians of multiple images obtained using filters with varying cutoff values. It then found the intersection point of all the Laplacians and placed the edge at that location. This technique was not verified, due to time constraints, but it is likely that it is at least as sensitive to noise as the Sobel and Canny algorithms. This edge detection technique also results in a longer analysis since the 3D image must be smoothed multiple times and the Laplacian calculated for each new smoothed image.

\subsubsection{Wall Motion Analysis}

Once the edge of the LV was obtained for each gate, the actual motion of the wall vs. time, for each LV sampling point, was obtained for use in phase analysis (section 2.2). Two different techniques were investigated for obtaining the wall motion used in phase analysis, time-activity curves and time-distance curves.

\section{Time Activity Curves}

Time-activity curves have been used historically in planar RNA phase analysis (45). It is an indirect method of calculating wall motion as it approximates the motion from the change in recorded counts as a function of time at fixed locations around the end-diastolic LV edge.

More specifically, circular regions of interest (ROI) $10 \mathrm{~mm}$ in diameter were positioned at each LV sampling point on the end-diastolic LV image (Figure 3.3a). As the heart beats, going from end-diastolic to end-systolic and back, these ROIs remained fixed 
and the total counts in each ROI was calculated for each gate. The change in counts vs. time is due to variable partial volume averaging along the edge of the LV and can be approximated by a sinusoidal function (Figure 3.3b). Time activity curves can be negatively affected by scatter, attenuation, and spill-over from adjacent organs, which may result in an incorrect reading of counts inside an ROI at a particular gate. This may be most problematic in the septal wall.

a)

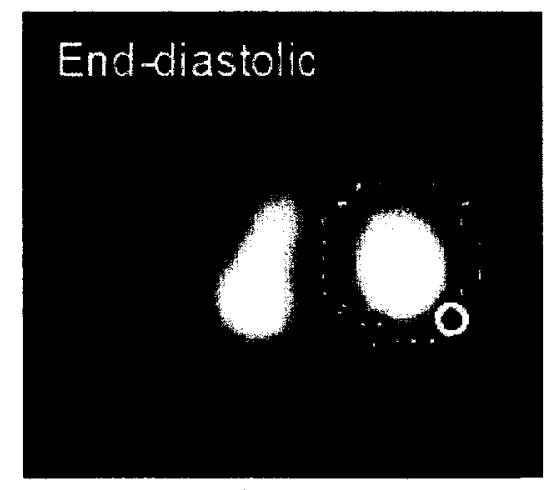

b)

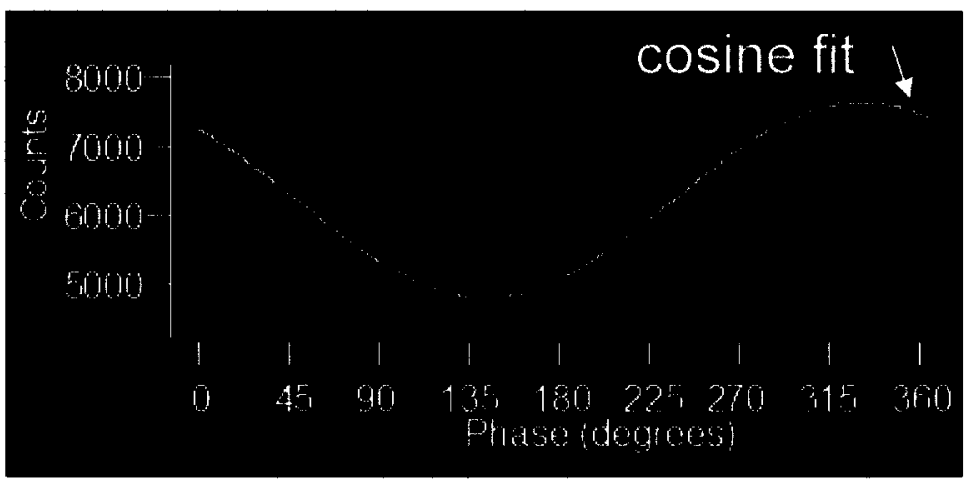

Figure 3.3: a) LV wall contour shown in green around the LV and a single sample circle is shown in white. All circles around the LV edge remain fixed as the heart beats, recording the change in counts. b) Time activity curve, shown in red, of the sample circle seen in a) with fitted cosine curve shown in yellow.

\section{Time Distance Curves}

A time distance technique measures wall motion directly since it tracks the LV edge as it contracts and translates during a heartbeat. For each LV sampling point, an edge was found at each gate. A vector was then drawn from a reference point to the edge. The length of the vector was then plotted vs. time (Figure 3.4a). Figure 3.4b, c, show an 
example of the LV edge detected at two different frames: end-diastolic and end-systolic. The graph shows the movement of a single LV sampling point, shown in white, on the LV edge with reference to the center of the LV. As with the time-activity curves, wall motion was approximated using a sinusoidal function (Figure 3.4a).

a)

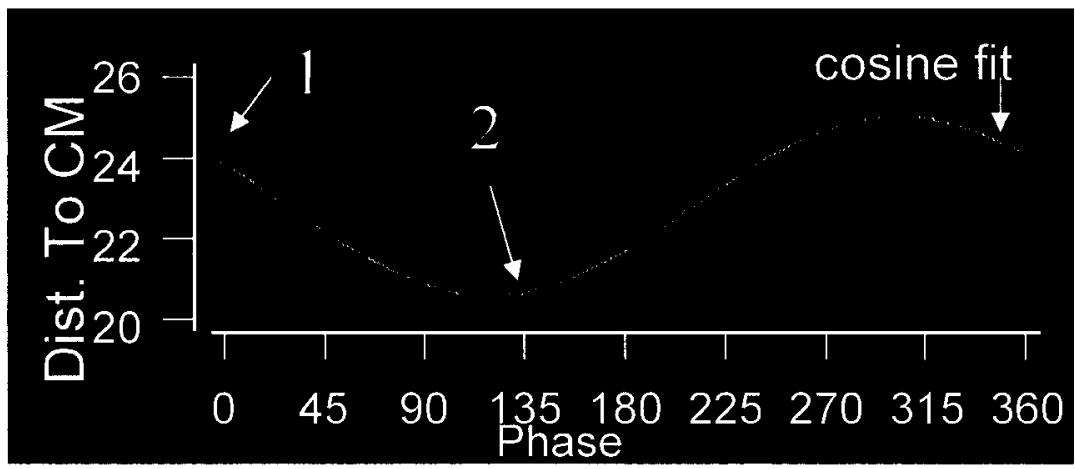

\section{End-diastolic (1) End-systolic (2)}

b)
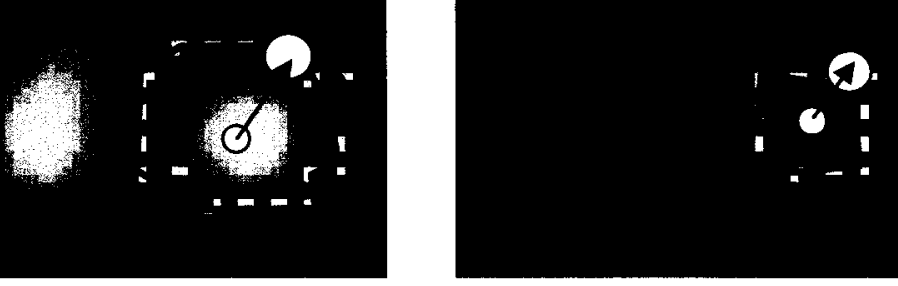

Figure 3.4: a) Time distance curve, shown in blue, of the sample LV edge data point with cosine fit shown in yellow. b) LV contour with sample edge data point (white circle) as it moves from end-diastolic gate, to $c$ ) end-systolic gate. The arrows represent the vector from the reference point to the LV edge data point. The length of this vector is used for further analysis. 
The length of the vector depends on the reference point. The reference point can be defined as either fixed or floating. A fixed reference point is user-defined inside the LV, along the long axis, and remains fixed through all gates. A floating reference point is calculated by finding the center of mass of the LV for each gate, thus giving each gate a different reference point. The possible advantage of a floating reference point is the ability to follow the LV if it shifts or translates. However, it may be possible that dyssynchrony could be misinterpreted as a translation if a floating reference point is used. Both of these time distance-based techniques, as well the time-activity technique, were investigated to determine which performed best at quantifying mechanical dyssynchrony.

\subsubsection{Polar Map Based Phase Analysis}

Once wall motion was obtained, using either time activity-based, time distance-based fixed or time distance-based float methods, the curves were fit to a cosine to obtain the phase and amplitude (section 2.2.1) for each LV sampling point and plotted in polar map format. Polar maps are a two-dimensional representation of the three dimensional LV surface. Recall that the edge detection is performed on 18 slices from apex to base. The LV edge of each slice is approximated to a circular ring for the polar map graphical representation, starting at the middle of the polar map (apex slices) through to the outer ring (base slices). Phase (Figure 3.5a) and amplitude (Figure 3.5b) values for each LV wall sampling point were then separately plotted in polar map format. Polar maps 
provide a visual map of regional phase and amplitude and may be useful in determining the site of latest activation using a 17-segment model (46) (Figure 4.5c).

a)

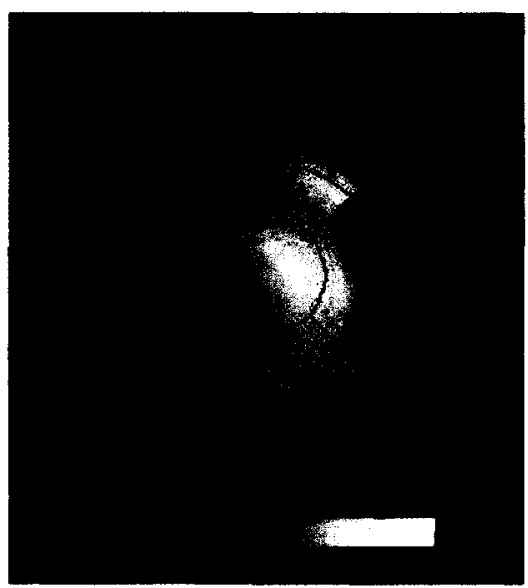

b)

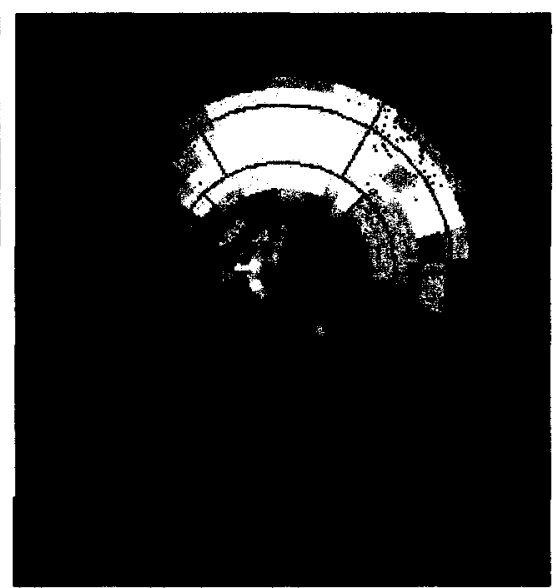

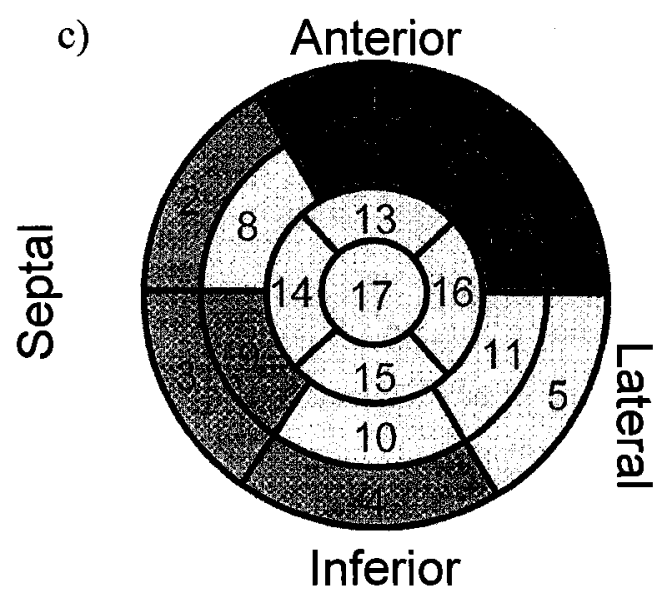

Figure 3.5: a) Sample phase polar map of patient with mechanical dyssynchrony and b) sample amplitude polar map with arbitrary color scale of same patient. c) Representation of 17 segment polar map model. The center of the polar map represents the apex of the LV. The outer rings of the polar map represent the basal rings. 


\subsection{Validation Study}

\subsubsection{Validation of the Quantification of Mechanical Dyssynchrony}

Initial validation of the SPECT RNA methods to quantify mechanical dyssynchrony described previously was done using the Normal and mechanical dyssynchrony populations. Receiver Operator Characteristic (ROC) analysis (see Appendix B) for the three LV sampling techniques (time activity, time distance fixed, time distance float) and the three phase analysis parameters (phase $\mathrm{SD}$, synchrony, entropy) was performed using ROCKIT software (University of Chicago) to determine the ability of the algorithms to detect mechanical dyssynchrony. ROC area, standard error of the ROC area and $95 \%$ confidence intervals were calculated for each combination of LV sampling technique and phase parameter.

\subsubsection{Variability}

Both normal and mechanical dyssynchrony population data sets were analyzed twice by the same operator (observer 1), and twice by two different operators (observer 1 and observer 2) to obtain intra- and inter-observer correlation coefficients for each combination of LV wall sampling technique (time activity, time distance float, time distance fixed) and phase parameter (phase SD, synchrony, entropy). Both operators were students trained by a professional technician. A linear regression of measurements and repeat measurements made by observer 1 was performed to obtain intra-observer 
correlation. A linear fit was then performed on the data to obtain slope and intercept values. A Bland-Altman plot was also created for analysis. Similarly, for inter-observer measurements, a linear regression and Bland-Altman plot were plotted to compare differences between observer 1 and observer 2 measurements for each LV sampling technique and parameter.

\subsubsection{Ability to Predict CRT Response}

ROC analysis was performed on 49 mechanical dyssynchrony patients to determine the ability of SPECT RNA phase analysis to predict response to CRT. ROC areas, standard errors of ROC area, and corresponding $95 \%$ confidence interval were calculated for each LV wall sampling technique and phase parameter. 


\section{Chapter 4: Results}

\subsection{Normal vs. Mechanical Dyssynchrony Population}

The results of the ROC analysis of the normal vs. mechanical dyssynchrony population are tabulated in Table 4.1 and the ROC curves are plotted in Figures 4.1, 4.2 and 4.3 for phase SD, synchrony, and entropy, respectively. Each figure depicts three ROC curves, one for each LV wall sampling technique: time activity, time distance float and time distance fixed.

Table 4.1: ROC areas for different combination of LV sampling methods and phase parameters for normal vs. mechanical dyssynchrony population. Standard error of the ROC area is presented in brackets.

\begin{tabular}{|c|c|c|c|}
\hline & Time activity & Time distance Float & Time distance Fixed \\
\hline phase SD & $1.00(\mathrm{n} / \mathrm{a})$ & $0.79(0.06)$ & $0.81(0.06)$ \\
\hline S & $1.00(\mathrm{n} / \mathrm{a})$ & $0.71(0.08)$ & $0.74(0.08)$ \\
\hline E & $0.99(0.01)$ & $0.80(0.07)$ & $0.85(0.06)$ \\
\hline
\end{tabular}




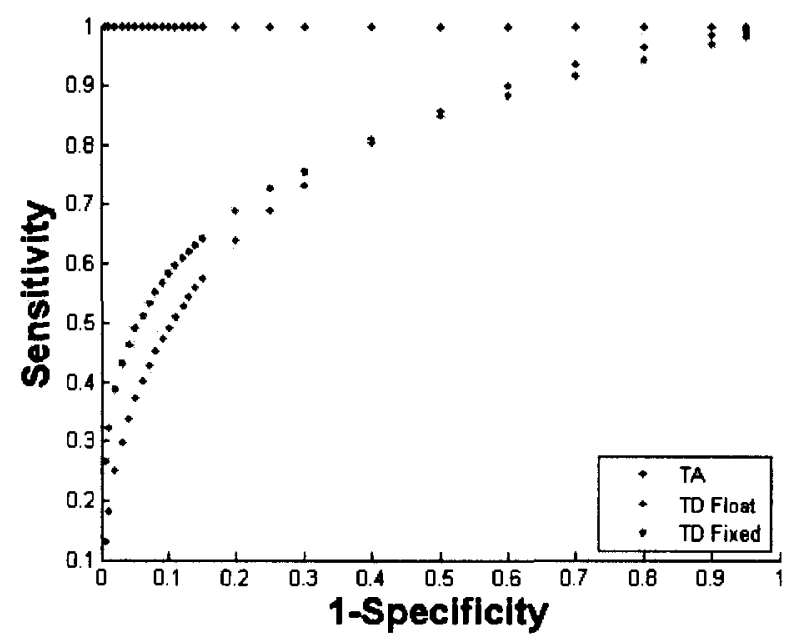

Figure 4.1: Phase SD ROC curves for normal vs. mechanical dyssynchrony population

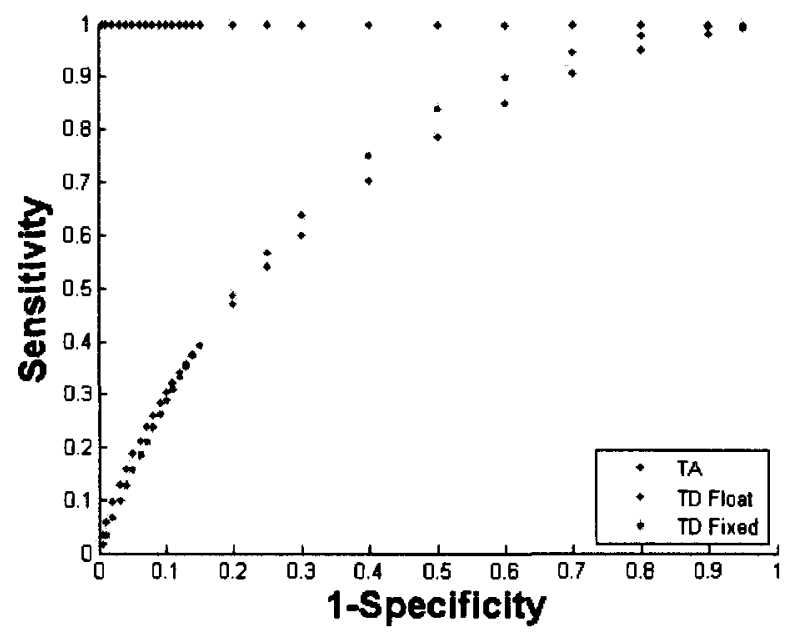

Figure 4.2: Synchrony ROC Curves for normal vs. mechanical dyssynchrony population 


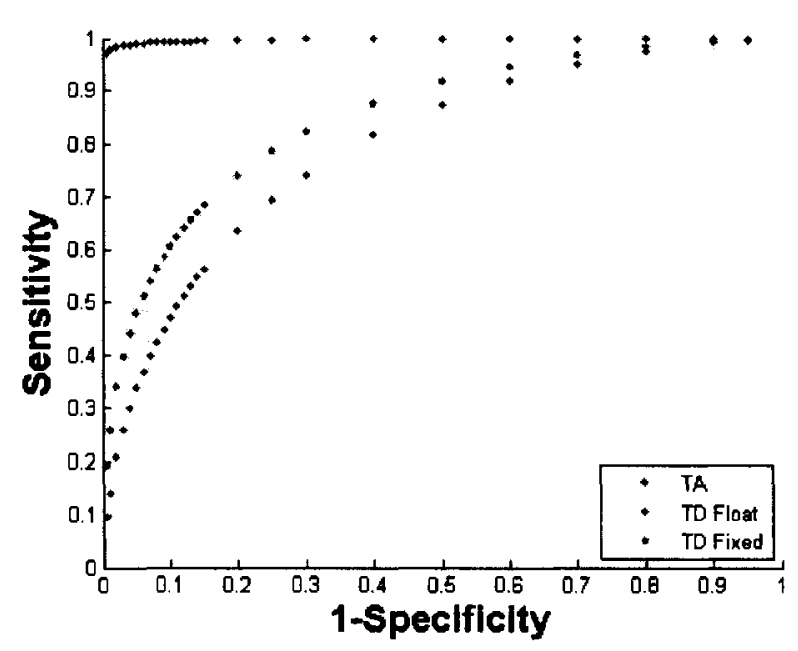

Figure 4.3: Entropy ROC Curves for normal vs. mechanical dyssynchrony population

The time activity-based technique showed excellent results with $\mathrm{ROC}$ areas near or at unity for all three parameters, outperforming both time distance-based techniques for all three parameters. For all three parameters, ROC areas obtained from the time activitybased technique were significantly better than both time distance-based techniques $(p<0.05)$. Differences between the two time distance-based techniques were not statistically significant for any single parameter. No error was calculated for the time activity-based synchrony and phase SD parameters due to the inability of the ROCKIT software to perform a full analysis when the data exhibited perfect separation.

An ROC area of 1.00 indicated that the time activity-based technique was able to perfectly discriminate between the normal and mechanical dyssynchrony Populations. A sensitivity and specificity of $100 \%$ was obtained at a cutoff value of $16^{\circ}$ for phase SD and 0.981 for synchrony. The ROC area for entropy was 0.99 , and at a cutoff value of 0.640 , sensitivity and specificity values of $98 \%$ were obtained. 
No significant differences $(\mathrm{p}<0.05)$ were found between the three time activity-based parameters. For both time distance-based techniques, only synchrony was found to be significantly different from the other two parameters $(\mathrm{p}<0.05)$.

The differences in phase SD between the normal and mechanical dyssynchrony populations were visually observed in the phase polar maps. Normal subject polar maps were largely uniform (low phase SD values) as seen in Figure 4.4b. Mechanical dyssynchrony patients, which had a larger phase SD compared to the normal population, all possessed a localized region of non-uniformity, often in the apical septal region, as seen in Figure 4.4a, rather than global heterogeneities.

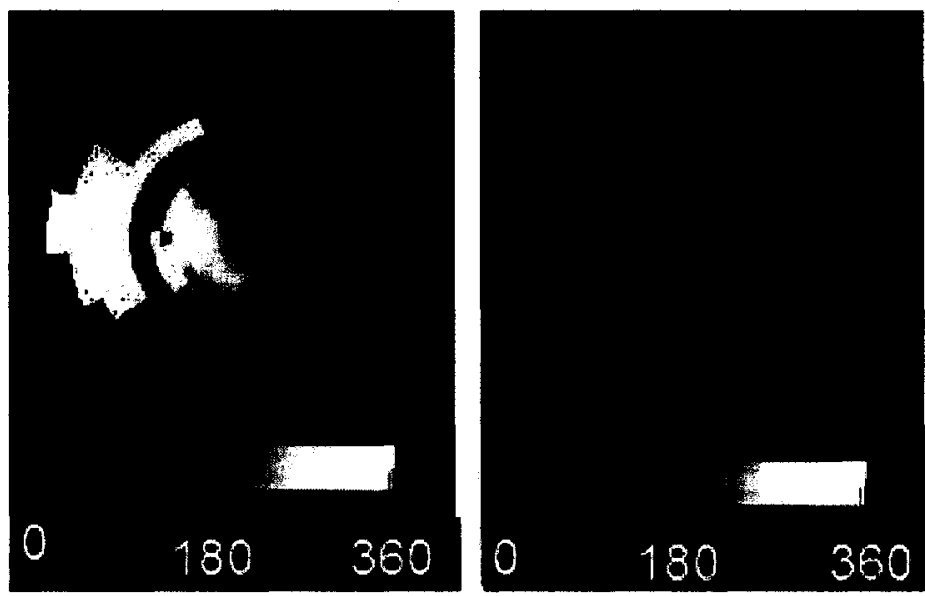

Figure 4.4: Phase polar map from a) a mechanical dyssynchrony patient with heterogeneous region around the septal wall and apex. b) a normal subject with uniform phase shown. 


\subsection{Observer Variability}

Intra-observer linear regression comparing two sets of measurements and a corresponding Bland-Altman plot are shown in Figure 4.5 for time activity-based phase parameters: phase SD (top row), synchrony (middle row), and entropy (bottom row). Correlation coefficients, $r$, are given in the linear regression graph along with slope and intercept values obtained from the linear fit. Similarly, Figures 4.6 and 4.7 show a linear regression and Bland-Altman plot of each phase parameter for time distance float and time distance fixed respectively. Figures $4.8,4.9$, and 4.10 show the inter-observer linear regression, Bland-Altman plot, correlation coefficient, fitted slope and intercept in an identical manner to those shown in the intra-observer graphs for time activity, time distance float and time distance fixed, respectively 

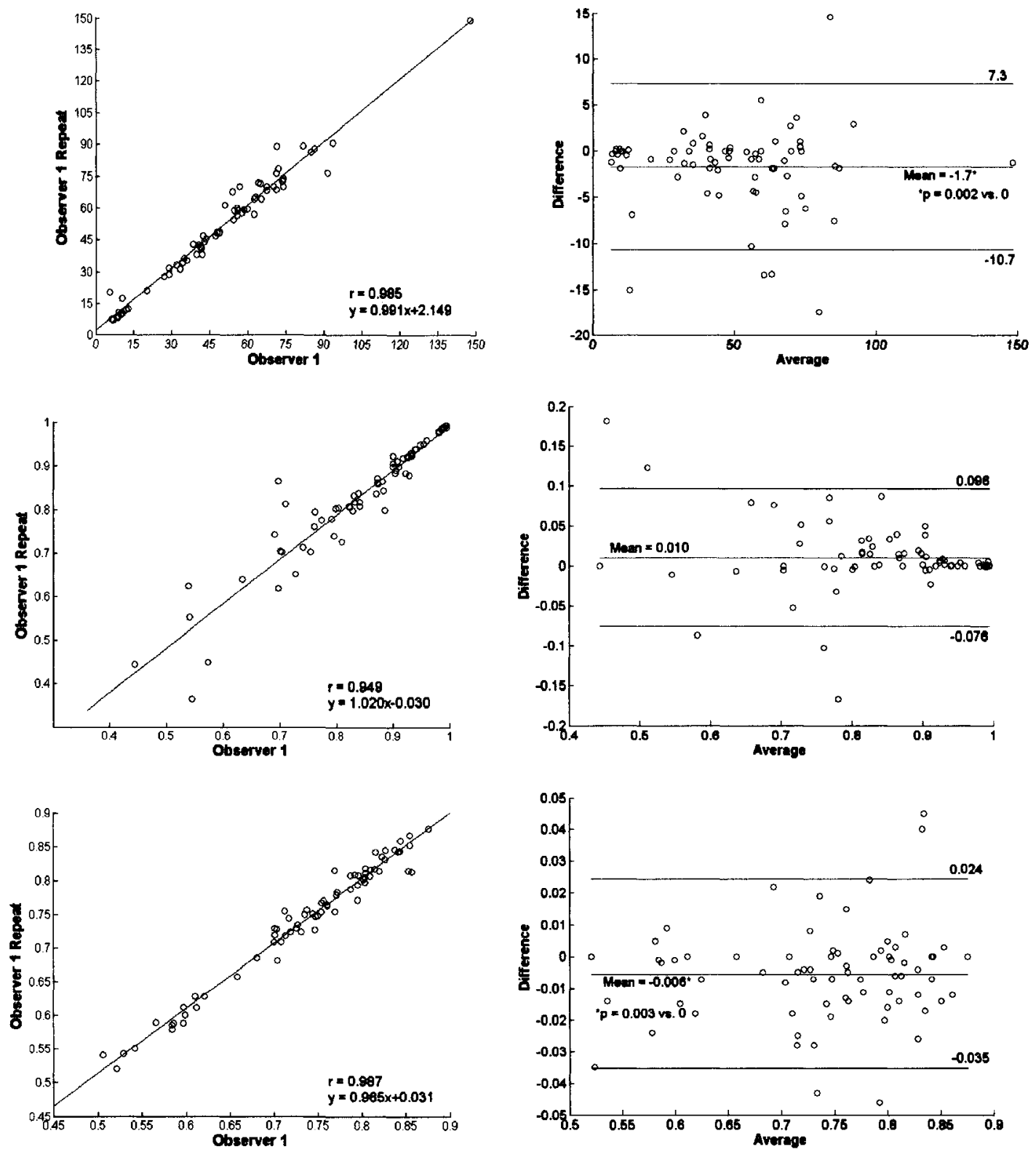

Figure 4.5: Time activity-based intra-observer regression analysis (left) and corresponding Bland-Altman plot (right) for phase SD (top row), synchrony (middle row), and entropy (bottom row). A correlation coefficient, $r$, as well as a slope and intercept obtained from a linear fit are given in the regression analysis. The red line in the Bland-Altman represents the mean value, and the blue lines represent the $95 \%$ confidence intervals of the data. 

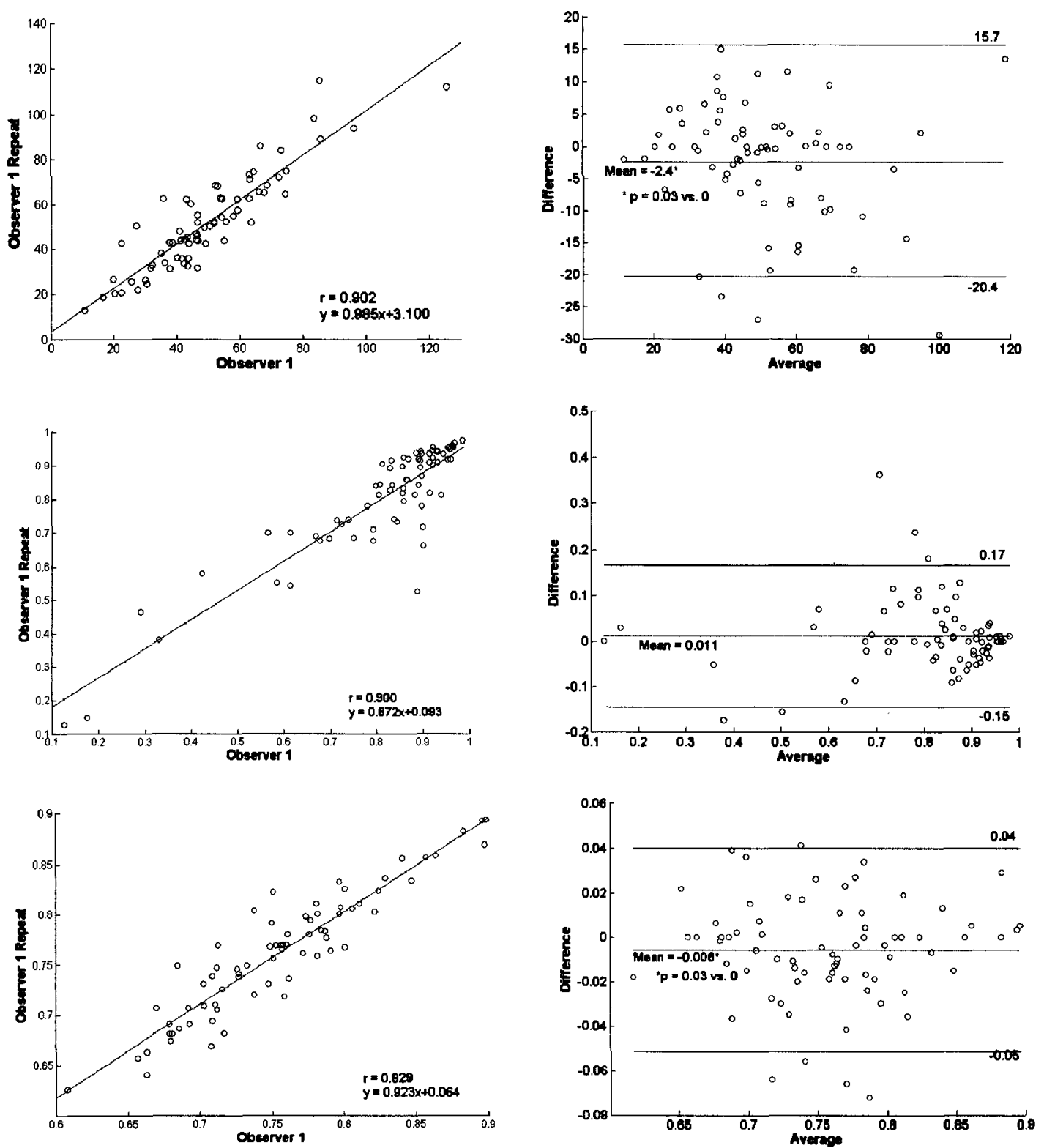

Figure 4.6: Time distance float-based intra-observer regression analysis (left) and corresponding Bland-Altman plot (right) for phase SD (top row), synchrony (middle row), and entropy (bottom row). A correlation coefficient, $r$, as well as a slope and intercept obtained from a linear fit are given in the regression analysis. The red line in the Bland-Altman represents the mean value, and the blue lines represent the $95 \%$ confidence intervals of the data. 

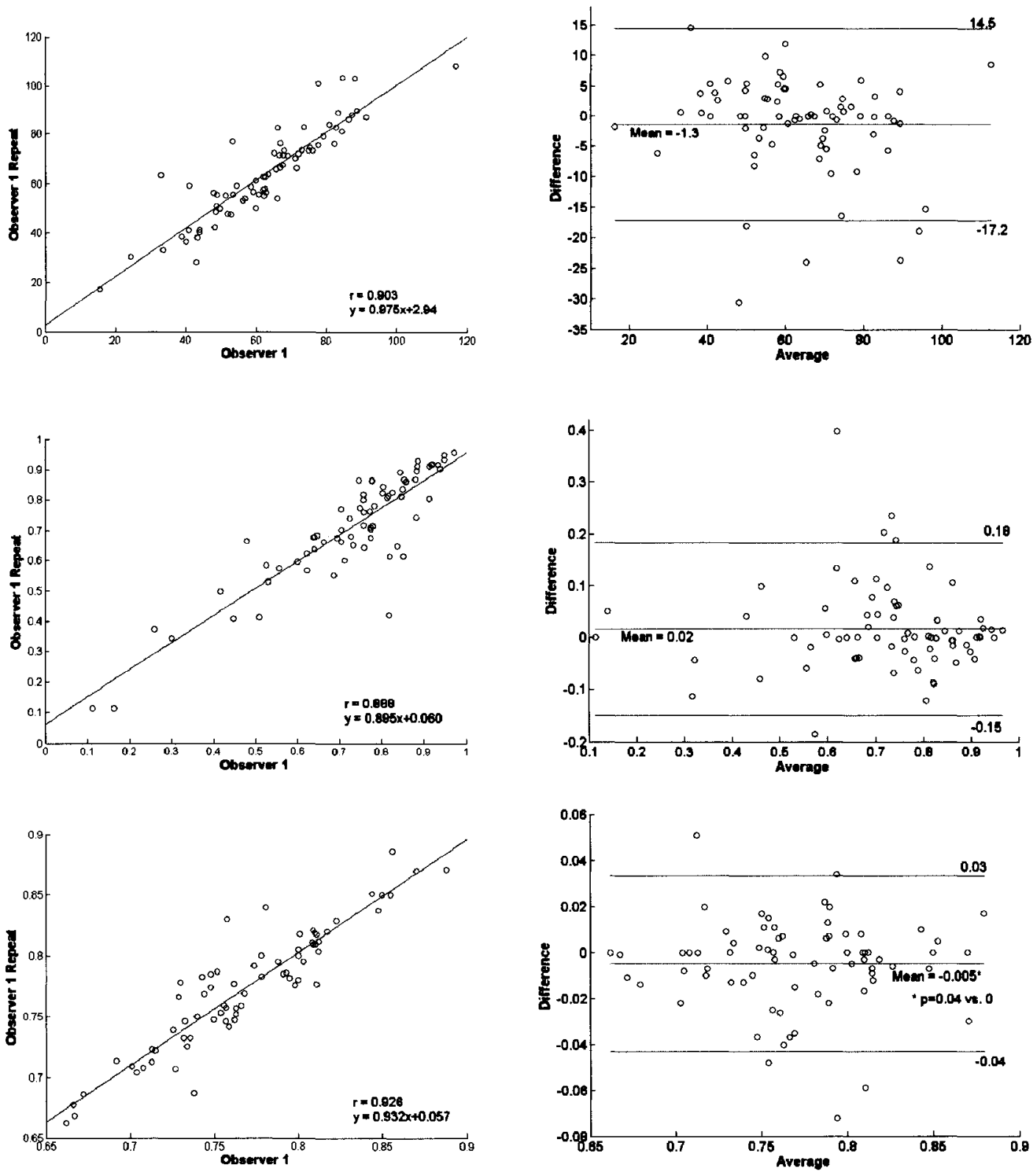

Figure 4.7: Time distance fixed-based intra-observer regression analysis (left) and corresponding Bland-Altman plot (right) for phase SD (top row), synchrony (middle row), and entropy (bottom row). A correlation coefficient, $r$, as well as a slope and intercept obtained from a linear fit are given in the regression analysis. The red line in the Bland-Altman represents the mean value, and the blue lines represent the $95 \%$ confidence intervals of the data. 

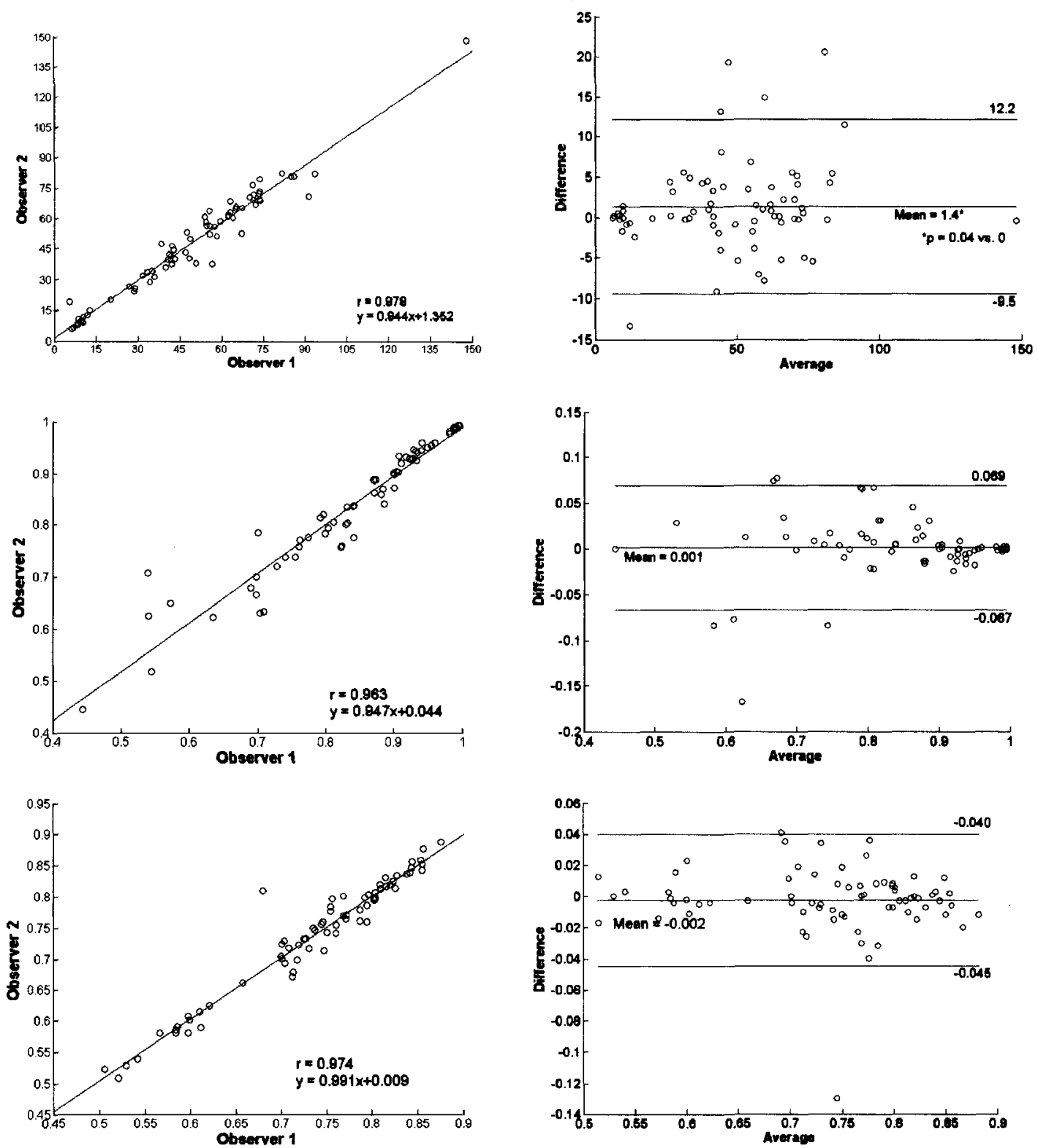

Figure 4.8: Time activity-based inter-observer regression analysis (left) and corresponding Bland-Altman plot (right) for phase SD (top row), synchrony (middle row), and entropy (bottom row). A correlation coefficient, $r$, as well as a slope and intercept obtained from a linear fit are given in the regression analysis. The red line in the Bland-Altman represents the mean value, and the blue lines represent the $95 \%$ confidence intervals of the data. 

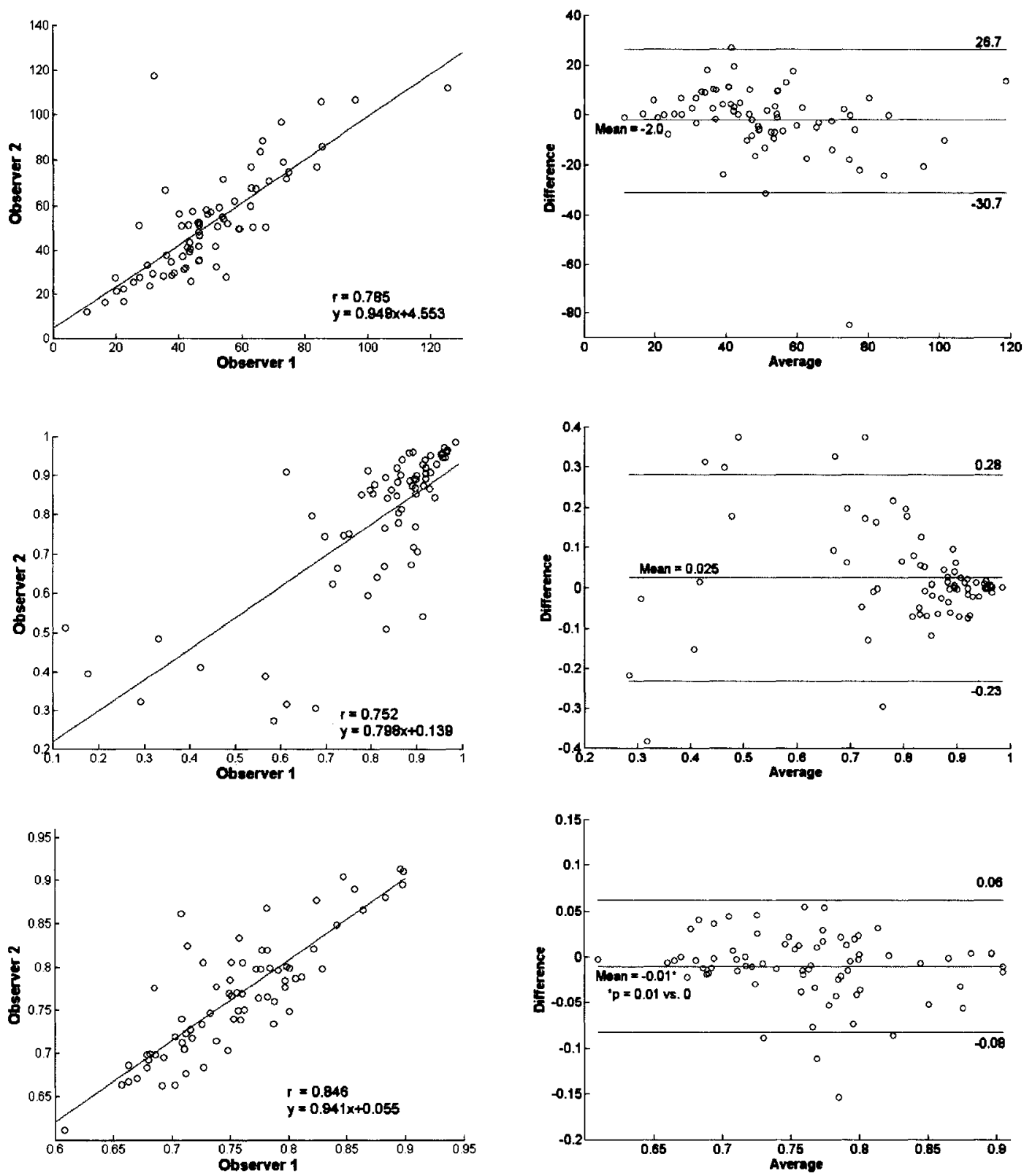

Figure 4.9: Time distance float-based inter-observer regression analysis (left) and corresponding Bland-Altman plot (right) for phase SD (top row), synchrony (middle row), and entropy (bottom row). A correlation coefficient, $r$, as well as a slope and intercept obtained from a linear fit are given in the regression analysis. The red line in the Bland-Altman represents the mean value, and the blue lines represent the $95 \%$ confidence intervals of the data. 

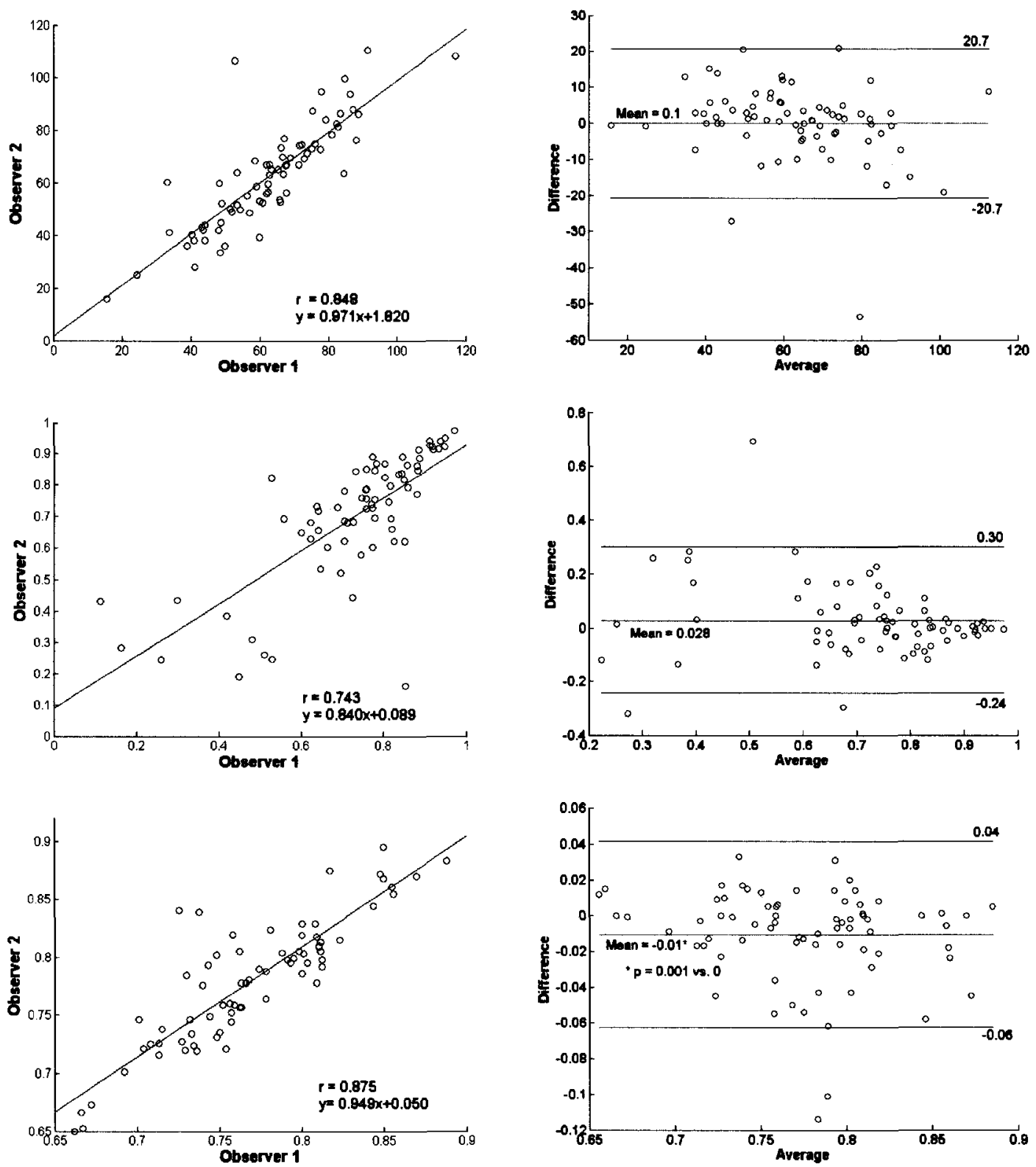

Figure 4.10: Time distance fixed-based inter-observer regression analysis (left) and corresponding Bland-Altman plot (right) for phase SD (top row), synchrony (middle row), and entropy (bottom row). A correlation coefficient, $r$, as well as a slope and intercept obtained from a linear fit are given in the regression analysis. The red line in the Bland-Altman represents the mean value, and the blue lines represent the $95 \%$ confidence intervals of the data. 
All time activity-based results produced excellent intra- and inter-observer correlation coefficient values of 0.94 or better for all three phase parameters. Time distance-based techniques displayed similar results to each other, with correlation coefficient values ranging from 0.88 to 0.93 for intra-observer values. Inter-observer correlation coefficients for synchrony were not as strong with values of 0.74 and 0.75 respectively for time distance fixed and float. The remaining time distance-based inter-observer correlation coefficients were comparable to those obtained for intra-observer. Synchrony mean difference values, obtained from Bland-Altman analysis, for all combinations of LV wall sampling techniques and intra- and inter-observer comparisons were not significantl y different from a zero mean $(\mathrm{p}<0.05)$, whereas entropy and phase SD mean difference values indicated significant differences from a mean of zero in at least half of the combinations. Table 4.2 presents the standard deviation of the mean difference over the mean parameter value of the parameter as obtained from analysis of Bland-Altman plots. Entropy values presented the lowest bias, under 5\% in all cases, whereas time activity based synchrony values presented biases close to 5\%. Remaining synchrony and phase SD values indicated biases ranging from $8 \%$ up to $28 \%$. 
Table 4.2: Observer bias expressed as the standard deviation of the mean difference over the mean parameter value (in percentage) obtained from Bland-Altman plots for each combination of LV wall sampling technique and phase parameter for both intra- and inter-observer values.

\begin{tabular}{|l|c|c|c|c|c|c|}
\hline \multirow{2}{*}{} & \multicolumn{2}{|c|}{ Intra-observer (SD / Mean Value) } & \multicolumn{3}{|c|}{ Inter-observer (SD / Mean Value) } \\
\cline { 2 - 7 } & Phase SD & Synchrony & Entropy & Phase SD & Synchrony & Entropy \\
\hline $\begin{array}{l}\text { Time } \\
\text { activity }\end{array}$ & $7.9 \%$ & $5.2 \%$ & $2.0 \%$ & $10.6 \%$ & $5.3 \%$ & $2.8 \%$ \\
\hline $\begin{array}{l}\text { Time } \\
\text { distance } \\
\text { float }\end{array}$ & $17.0 \%$ & $10.3 \%$ & $3.0 \%$ & $28.0 \%$ & $16.5 \%$ & $4.7 \%$ \\
\hline $\begin{array}{l}\text { Time } \\
\text { distance } \\
\text { fixed }\end{array}$ & $12.1 \%$ & $11.7 \%$ & $2.3 \%$ & $16.3 \%$ & $19.7 \%$ & $3.3 \%$ \\
\hline
\end{tabular}

\subsection{CRT Response}

The ROC area results of the analysis for prediction of CRT response are presented in Table 4.3.

Table 4.3: ROC Areas for different LV sampling methods for prediction of responders in mechanical dyssynchrony population. Standard error of ROC area is presented in brackets.

\begin{tabular}{|c|c|c|c|}
\hline & Time activity & Time distance float & Time distance fixed \\
\hline Phase SD & $0.69(0.08)$ & $0.69(0.08)$ & $0.71(0.07)$ \\
\hline S & $0.75(0.07)$ & $0.56(0.08)$ & $0.58(0.08)$ \\
\hline E & $0.68(0.08)$ & $0.69(0.07)$ & $0.72(0.07)$ \\
\hline
\end{tabular}


Figures $4.11,4.12$, and 4.13 depict the ROC curves, one for each LV wall sampling technique: time activity, time distance float and time distance fixed.

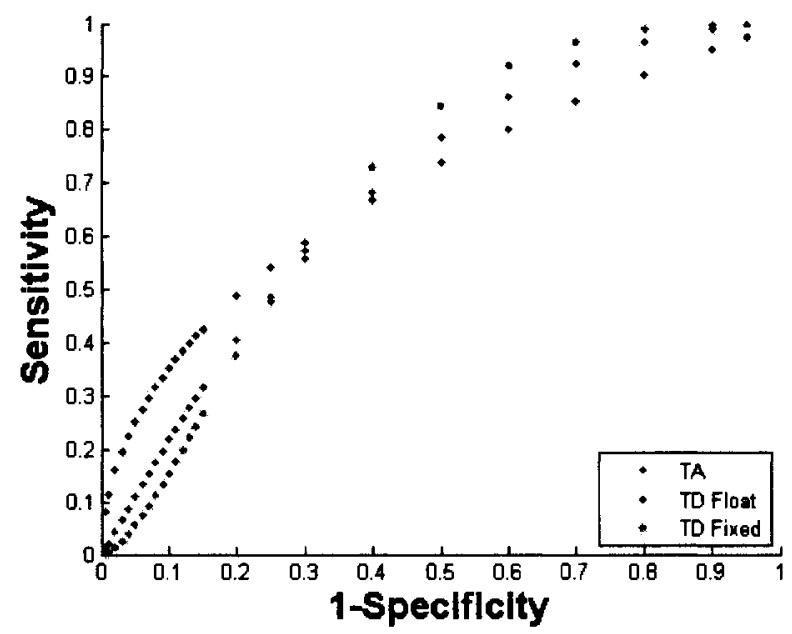

Figure 4.11: ROC curves for phase SD for prediction of CRT responders

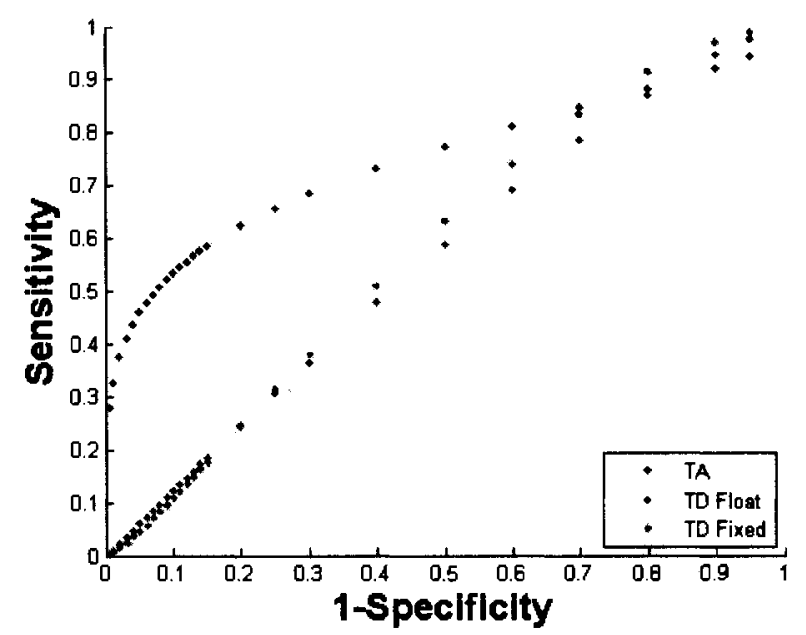

Figure 4.12: ROC curves for synchrony for prediction of CRT responders 


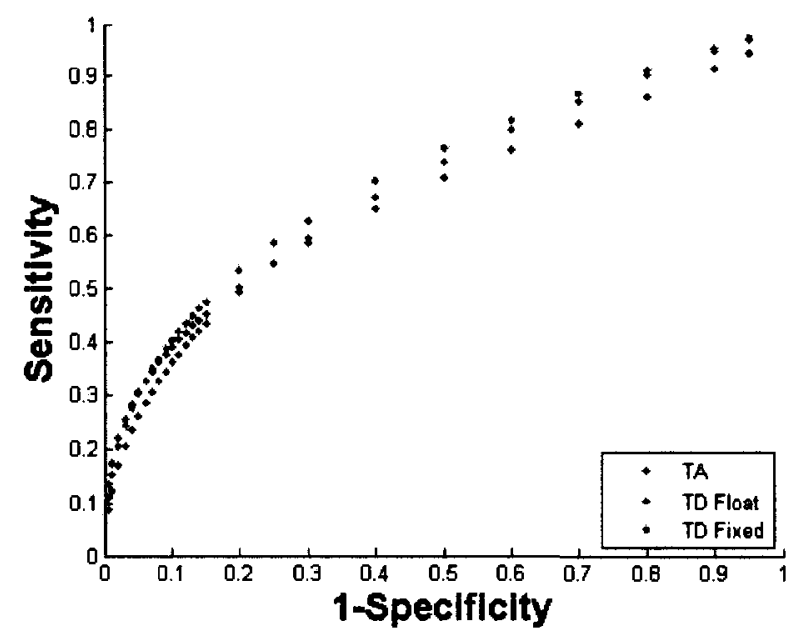

Figure 4.13: ROC curves for entropy for prediction of CRT responders

All 3 wall motion techniques showed similar ROC area results varying between 0.68 and 0.72 for phase SD and entropy. Results indicate time activity-based synchrony performed best with an ROC area of 0.75 , which was statistically different from time distance float based synchrony $(\mathrm{p}<0.05)$ but not time distance fixed based synchrony $(\mathrm{p}=0.06)$. Time activity-based synchrony was not significantly different from the other time activity-based parameters $(\mathrm{p}>0.05)$. None of the ROC area differences between time activity-based and time distance-based techniques were statistically significant for phase $\mathrm{SD}$ and entropy $(\mathrm{p}>0.05)$. Using the time activity-based parameters, sensitivity and specificity values of $69 \%$ and $68 \%$ were obtained for synchrony at a cutoff of 0.849 . The cutoff was chosen where sensitivity and specificity were nearly equal. Similarly, at a cutoff of $55.2^{\circ}$, time activity-based phase SD sensitivity and specificity values were $65 \%$ and $62 \%$ respectively. For time activity-based entropy, sensitivity and specificity values of $64 \%$ and $61 \%$ values were obtained at a cutoff of 0.779 . 
Figure 4.14 presents box-plots of the parameter values for responders and nonresponders. Results indicated that responder time activity-based phase parameter values were closer to normal values than those of the non-responders. This was, however, only significant for synchrony $(\mathrm{p}<0.05)$.

a)

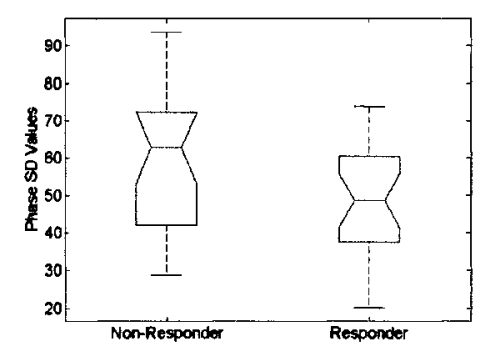

b)

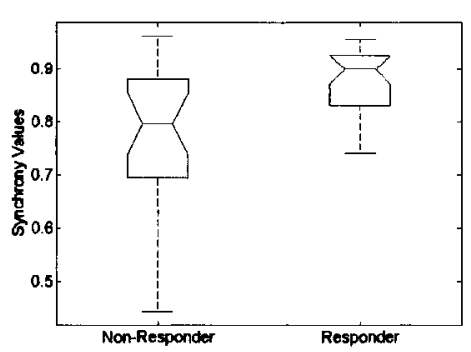

c)

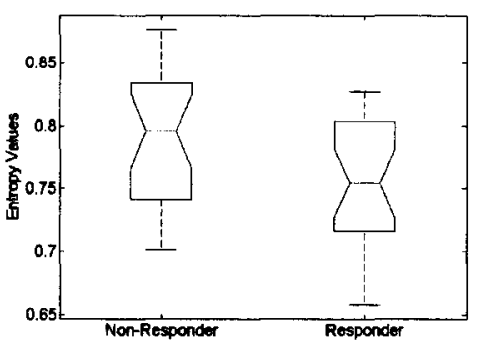

Figure 4.14: Notched (representing 95\% confidence intervals) box-plot comparison of responders and non-responders for a) phase SD, b) synchrony, c) entropy of time activity-based analysis.

While every phase polar map displayed a region of non-uniformity, no visual trends or specific locations of non-uniformity were found to separate responders from nonresponders. 


\section{Chapter 5: Discussion}

In this thesis, SPECT RNA phase analysis methods for quantifying mechanical dyssynchrony were investigated. Techniques of measuring LV wall motion for use in quantifying mechanical dyssynchrony were developed and validated. The ability of SPECT RNA phase analysis at predicting CRT response was determined. Correlation coefficients for intra- and inter-observer variability were also measured.

The time activity-based phase analysis was found to perform equally as well as or significantly better than either time distance-based techniques at detecting mechanical dyssynchrony. One of the reasons for this may be the fact that time activity curves were less susceptible to errors in the edge detection. The time distance-based techniques attempted to follow the actual movement of the LV wall during cardiac contraction. It had to therefore perform edge detection on each and every gate, increasing the possibilities of error due to misinterpretation and poor edge detection. The time activitybased method only required edge detection at the end-diastolic gate, and since a large ROI was used, the time activity-based method may potentially be less sensitive to small changes in its initial location. 
Time distance-based synchrony was found to have a significantly lower ROC area compared to time distance-based entropy and phase SD. Since synchrony is the only parameter which takes amplitude into account, this result suggests that the calculation of amplitude using time distance-based methods is not accurate.

It has also been suggested by Henneman et al. in an editorial response (47), that a time activity-based technique may account for all three types of cardiac movements occurring during contraction: rotation, translation, and contraction itself. These motions would all affect the time activity curves in a given ROI, and would thus be included in the analysis. Conversely, both of the time distance-based methods used in this thesis ignored rotational effects, while time distance fixed curves also did not account for translation. This may be another explanation for the poorer results obtained using time distance-based methods. A recent paper (48) investigated a technique called optical flow motion analysis, which was able to accurately track the LV wall in four dimensions (spatial and temporal). Though more complex and difficult to implement, it may be possible for time distance-based techniques to track rotation as well as contraction and translation using optical flow motion analysis, which may improve CRT response prediction.

The SPECT RNA results obtained using time activity-based methods were compared to planar RNA phase analysis results (Table 5.1) obtained in our lab, but not as part of this thesis. SPECT RNA ROC areas were higher than those of planar RNA but were not significantly different at the $95 \%$ level. This can be explained by the relative ease required to differentiate between a normal heart and one with mechanical dyssynchrony. 
The marked differences between these two populations were visualized from the polar maps and phase histograms created. Phase polar maps for mechanical dyssynchrony patients possessed heterogeneous regions and their phase histograms were much broader, whereas normal populations possessed near homogeneous polar maps and narrow histogram distributions. This is confirmed by the three parameters, which for the majority indicated a clear division separating the two groups. However, the majority of the heterogeneous regions of mechanical dyssynchrony patients were found to be localized in the septal and apical regions. It was expected that the lateral wall would be most delayed in LBBB patients since a blockage would create dyssynchronous behaviour in LV regions which follow the location of the blockage. It is unclear at this time why the lateral wall of the patients did not appear delayed based on phase analysis.

Table 5.1: Comparison of ROC area results obtained from planar RNA, obtained in our lab and SPECT RNA for detecting mechanical dyssynchrony.

\begin{tabular}{|r|c|c|c|c|}
\hline & ROC Area & Sensitivity & Specificity & Cutoff \\
\hline Planar RNA & \multicolumn{5}{|l|}{} \\
\hline phase SD & 0.95 & 0.91 & 0.89 & $56^{\circ}$ \\
\hline S & 0.98 & 0.95 & 0.95 & 0.74 \\
\hline E & 0.99 & 0.94 & 0.96 & 0.75 \\
\hline SPECT RNA & \multicolumn{5}{|l}{} \\
\hline phase SD & 1.00 & 1.0 & 1.0 & $16^{\circ}$ \\
\hline S & 1.00 & 1.0 & 1.0 & 0.98 \\
\hline E & 0.99 & 0.98 & 0.98 & 0.64 \\
\hline
\end{tabular}


It is interesting to note, however, that while both planar RNA and SPECT RNA time activity methods presented near equal sensitivity and specificity, the corresponding cutoff values suggested by both methods differed considerably. Optimal SPECT RNA time activity-based cutoff values were $16^{\circ}, 0.981$ and 0.64 for phase SD, synchrony and entropy respectively. Those for planar RNA were $56^{\circ}, 0.76$ and 0.75 respectively. In all cases, the SPECT RNA time activity-based cutoff values were closer to ideal normal values. This could be explained by the fact that overlapping structures on a planar image may have caused a greater variety of phases to be present within the LV of normal patients. With SPECT RNA, there is no overlap and the values of the normal population reach the expected values more closely. This would suggest that SPECT RNA time activity-based parameter values are more accurate than those given by planar RNA.

The ROC areas for both time activity-based phase SD and time activity-based synchrony were calculated to be 1.0 , indicating perfect separation of the two groups. This is believed to be an artefact arising from the small size of the normal population $(\mathrm{N}=13)$. Increasing the size of the normal population would be expected to lead to a larger spread in the data and ROC areas below 1.00. However, the ROC areas are not expected to be decreased by much since the phase parameter values for the normal population was well defined with a small spread and good separation from the mechanical dyssynchrony population. This is seen in the high planar ROC values in Table 5.1, which were calculated using a normal population of $\mathrm{N}=36$.

High reproducibility of the phase parameters is necessary so that consistent results can be produced between centres. One of the potential strengths of SPECT RNA phase 
analysis is the automation of the analysis, thus reducing potential operator errors. Excellent correlation coefficient values for time activity-based phase, synchrony and entropy were observed. The correlation coefficients of time distance-based float and fixed techniques for both inter- and intra-observer measurements ranged from 0.85 to 0.93 , indicating good reproducibility though not as high as the time activity-based results. Time distance-based fixed and float synchrony correlation coefficients and time distancebased float phase SD indicated poor reproducibility with values under 0.800 . Synchrony mean difference values, obtained from Bland-Altman plots, for all three LV wall sampling techniques were found to not be different from zero for both intra- and interobserver reproducibility based on the Bland-Altman plots. Phase SD and entropy both produced Bland-Altman mean difference values significantly different from zero $(\mathrm{p}<0.05)$ for time activity based intra-observer reproducibility. Similarly, for time activity based inter-reproducibility, phase SD mean difference value was different from zero indicating a slight constant systematic difference between repeat measurements. Table 4.2 showed the presence of observer bias in certain combinations of LV wall sampling techniques and phase parameters by determining the ratio of standard deviation of the mean difference over the mean parameter value. However, the effect of this bias is believed to be relatively small since the ratio of the mean difference over the mean parameter value for all possible combinations was below $5 \%$. The confidence interval, and consequently the standard deviation of the mean, is relatively small, suggesting that the non-overlap with a zero mean is due to a tight distribution of the data. 
The time activity results are consistent with SPECT MPI phase analysis results. High correlation coefficients $(r>0.99)$ for SPECT MPI phase parameters, for both inter- and intra- observer measurements have been reported (6). Both the SPECT RNA (this thesis) and SPECT MPI perform semi-automated phase analysis, which allows for low intra- and inter-observer variability. In contrast, echocardiographic tissue Doppler imaging requires extensive operator input, which can be expected to result in larger inter- and intraobserver variability. This was the findings of the PROSPECT study (7), who reported high intra- and inter-observer variability values ranging from $11 \%$ to $72 \%$ for the various echocardiographic-based measures of mechanical dyssynchrony. Following the same procedure used in the PROSPECT study, variability values were obtained for the results of this thesis, in order to properly compare with the results of the PROSPECT. SPECT RNA intra- and inter-observer variability values produced excellent results of $5.5 \%$ or better for all phase parameters using time activity-based analysis. The ability to perform semi-automated analysis, thus reducing observer variability, is a strength of SPECT RNA phase analysis.

The prediction of CRT response did not produce the same quality of results that were observed for the normal vs. mechanical dyssynchrony study. This was not completely unexpected as there are smaller differences in cardiac dyssynchrony between responders and non-responders than compared to normal and mechanical dyssynchrony patients. Though not statistically significant, time activity-based synchrony performed the best out of all combinations of $\mathrm{LV}$ wall motion techniques and phase parameters with an ROC area of 0.75 and a sensitivity and specificity of $69 \%$ and $68 \%$ respectively. The results 
also suggested that responders tended to be the patients whose synchrony, entropy and phase SD values approached the expected normal values. Non-responder parameter values were further away suggesting that severity of mechanical dyssynchrony may impact chances of response. Table 5.2 presents a comparison of this work to other major published works attempting to predict CRT response.

The echocardiographic PROSPECT study $(7,27)$ investigated the predictive value of a variety of parameters for CRT response. The results of the PROSPECT study indicated that the septal-posterior wall motion delay (SPWMD) parameter performed the best out of all investigated, obtaining a ROC area of 0.62 . SPECT perfusion CRT responder prediction data from Henneman et al. (32) is also presented in Table 5.2. Phase SD was the parameter that performed the best from that study, producing an ROC area of 0.81 . The authors suggested using a cutoff of 43 degrees, leading to equal sensitivity and specificity values of $74 \%$. None of the published ROC areas were significantly different than those obtained in this study. It is also worth noting that the SPECT perfusion study used a minimum improvement of 1 level in NYHA score as a definition of response. This is a subjective measurement (1) and to properly compare these two techniques, it would be necessary to utilize the same definition of response.

SPECT RNA results obtained in this thesis and the other studies compared in Table $5.2(7,32)$, indicate that accurate quantification of mechanical dyssynchrony can be obtained. However, results from prediction to CRT response by these studies suggest that the quantification of mechanical dyssynchrony may not be the best predictive 
parameter of CRT response, and that other alternative measures, such as scarring and CRT lead placement should be investigated.

Table 5.2: Comparison of prediction of CRT response of SPECT RNA time activitybased synchrony (this thesis), the PROSPECT echocardiography study (7) and the SPECT perfusion study (32). The parameters that performed best from each study are shown in the table.

\begin{tabular}{|l|c|c|c|c|c|}
\hline & Parameter & $\begin{array}{c}\text { ROC } \\
\text { Area }\end{array}$ & Cutoff & $\begin{array}{c}\text { Sensitivity / } \\
\text { Specificity }\end{array}$ & $\begin{array}{c}\text { Population } \\
\text { Size }\end{array}$ \\
\hline SPECT RNA (this work) & S & 0.75 & 0.981 & $69 \% / 68 \%$ & 49 \\
\hline PROSPECT study & SPWMD & 0.62 & $30 \mathrm{~ms}$ & $64 \% / 52 \%$ & 426 \\
\hline SPECT Perfusion & Bandwidth & 0.78 & $135^{\circ}$ & $70 \% / 70 \%$ & 42 \\
\hline SPECT Perfusion & phase SD & 0.81 & $43^{\circ}$ & $74 \% / 74 \%$ & 42 \\
\hline
\end{tabular}




\section{Chapter 6: Future Directions}

Phase polar map heterogeneities in the mechanical dyssynchrony population were qualitatively assessed, but no obvious trends or differences were discovered between the responder and non-responder groups. A more-in depth quantitative analysis of the regions is warranted in order to determine whether any heterogeneous patterns within localized regions occurred, which may help predict CRT response.

It has been stipulated that placing the pacemaker lead at the site of latest activation (i.e. largest phase value) may increase the chance of response. The next step in the analysis would be to determine if there is any correlation between site of latest activation, lead placement, and response. The data acquired in this study includes the average phase values for the 17 segments of the polar map for each patient, pre- and post-CRT. It is therefore possible to determine whether the pre-CRT site of latest activation becomes the site of earliest activation in the post-CRT follow-up scan. Patients with matched sites of latest and earliest activation would be expected to have higher CRT response rates.

If the pacemaker lead is placed on scar tissue, a lower chance of response may be expected. The ability of SPECT perfusion to determine regions of scarring in the LV wall combined with SPECT RNA phase analysis developed in this work could be fused 
together in a polar map format to determine if scarring and sites of latest activation overlap. This could explain why some patients do not properly respond to CRT.

In this study, a reduction in LV end-systolic volume of $15 \%$ was the only definition of response used. However, it is not clear that this definition of response actually translates into improved patient outcome. Investigating different definitions of response may be worth pursuing. This includes comparing other remodelling criteria (improvement in LVEF) to subjective clinical capacity variables (improvement of NYHA score), soft-clinical end-points (subsequent hospitalizations due to $\mathrm{CHF}$ ), and hard clinical end-points (death or transplant). Within our lab, there is an ongoing CRT study with 3 year follow-up SPECT RNA scans. The data from this study can be analysed using the methodologies developed in this thesis to investigate the role that the definition of response has on the results presented in section 5.3 


\section{Chapter 7: Conclusion}

A semi-automated SPECT RNA phase analysis program capable of quantifying mechanical dyssynchrony was successfully developed and validated. Three methods of assessing wall motion were investigated. The time activity-based method was the least sensitive to errors in edge detection and was determined to provide the most robust measure of LV wall motion. All three phase parameters were found to have high ROC areas for diagnosing mechanical dyssynchrony. ROC areas for prediction of CRT response were significantly lower than the results of the normal vs. mechanical dyssynchrony analysis, but were comparable to other imaging modalities results. SPECT RNA presented excellent intra- and inter-observer correlation coefficients. Further work into assessing the optimal pacing site may allow the clinical use of SPECT RNA phase analysis for assessing CRT. Modest results comparable to previously published data from other imaging techniques suggests that mechanical dyssynchrony may not be a significant predictor of CRT response. 


\section{Appendix A: Software Development}

There currently exists no commercial software capable of performing SPECT RNA phase analysis, so as a major part of this thesis work, an in-house software application was developed. The program was created in a working collaboration with Hermes Medical Solutions, who provided hardware support, and was written in $\mathrm{C}++$ with the QT application. The program employs a semi-automated edge detection technique, described in section 3.4, followed by the automatic implementation and calculation of phase analysis, described in section 2.2 .

With this software, it is possible to visualize any combination of slice and gate in any of three orientations: short axis (SA), Horizontal Long Axis (HLA), and Vertical Long Axis (VLA) (Figure A.1). It is also possible to view a cine of the gated images in all of these orientations.

Sections of the screenshots are circled and numerically labelled to help understand the process. Once a patient's data is loaded into the program, the user chooses a SA slice (Figure A.1-(1)) where the LV and RV are visible and easily distinguishable. The user then positions a crosshair inside the LV on the SA, Figure A.1-(2). This determines the corresponding long axis images to be displayed. The user can then clearly see the LV in all three orientations. 


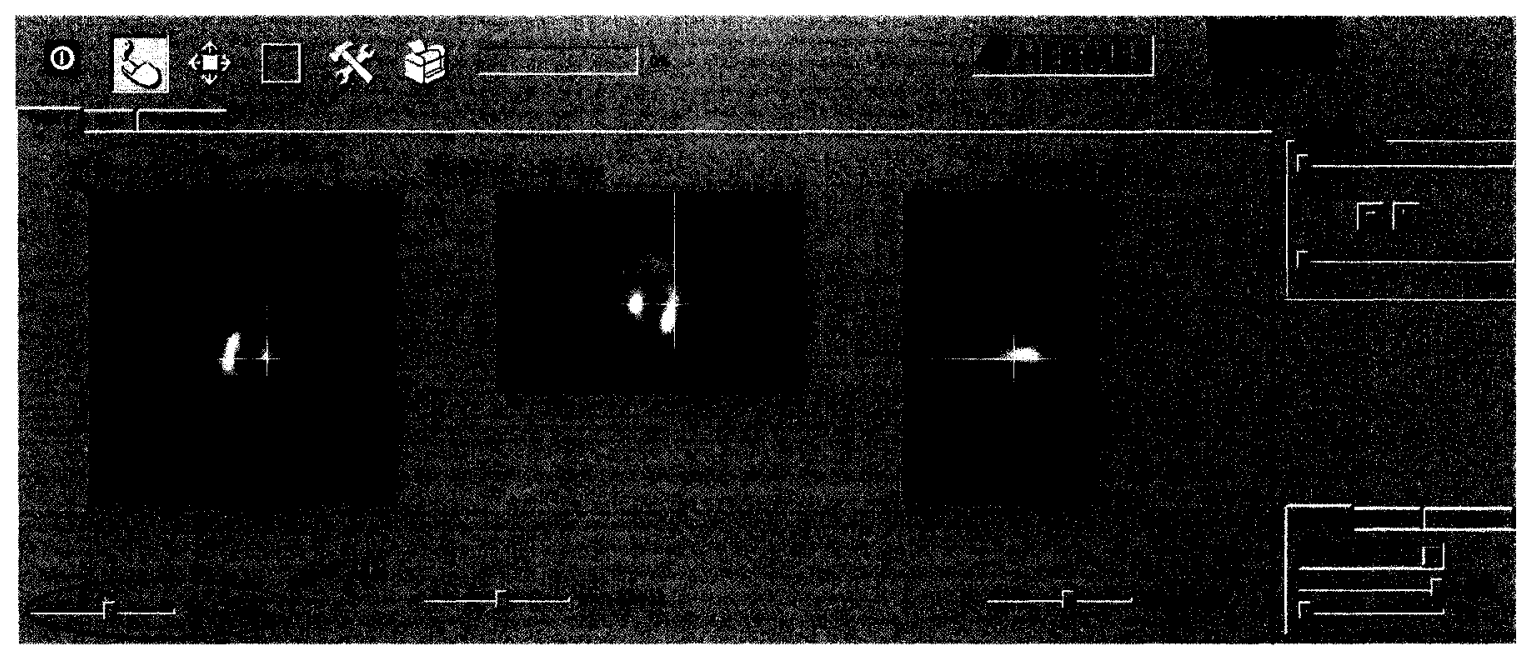

Figure A.1: Screenshot of program where user has defined a crosshair. The crosshair helps locate the corresponding HLA and VLA images facilitating further analysis.

Using the HLA and VLA slices, a box is positioned around the LV (Figure A.2-(3)). The ends of this user-defined box determine the location of the base and apex used to sample the LV wall (section 3.4). A blue line, defining the position of the fixed reference point, must also be positioned by the user. The user can adjust a color scale to assist in defining the location of the LV base and apex (Figure A.2-(4)). 


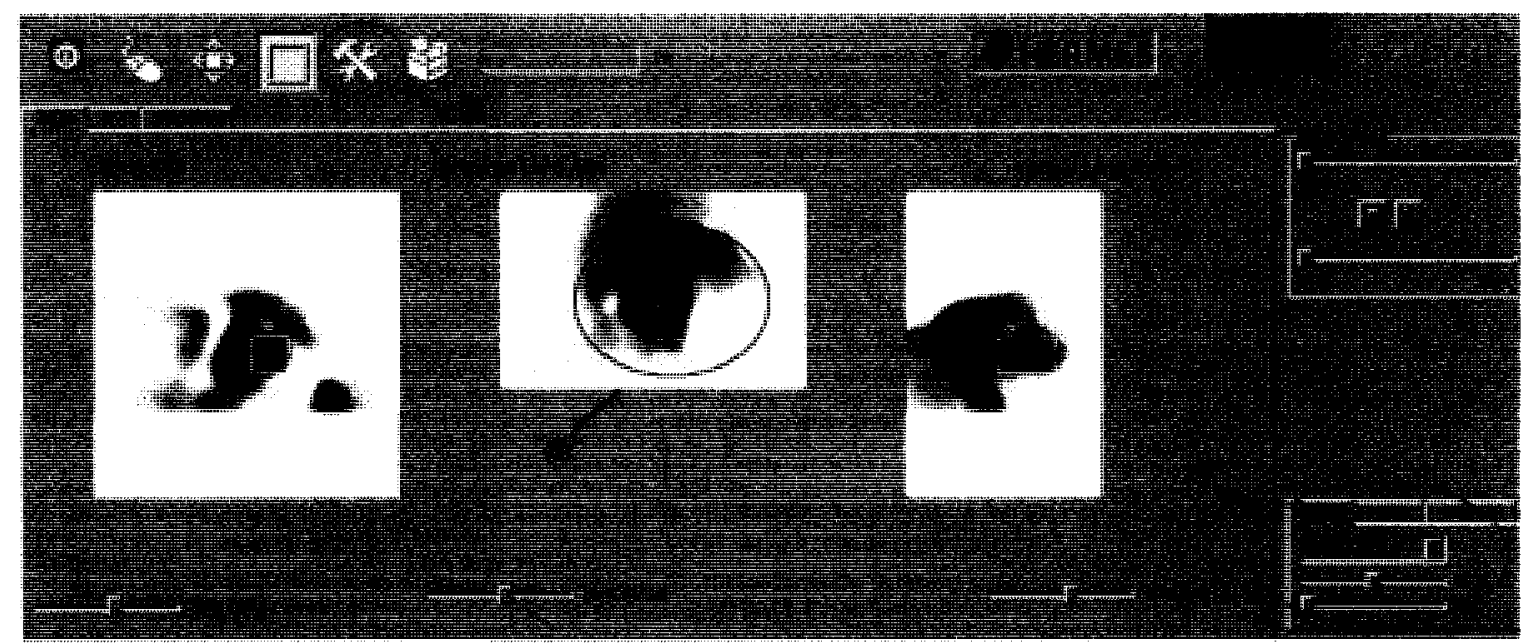

Figure A.2: Screenshot of program where user has changed color scheme and threshold value to facilitate positioning of the box around LV edge

Once the box is properly positioned, the user presses the tool button at the top of the interface (Figure A.3-(5)) and the LV edge is detected automatically by the program based on the LV box position. Afterwards, polar map-based phase analysis is performed and results for both time distance-based and time activity-based methods and all three phase parameters are calculated and displayed.

As discussed in section 3.4.1, the $\mathrm{LV}$ is resampled into $18 \mathrm{SA}$ slices using a canonical/cylindrical sampling algorithm. By clicking on the ROI tab (Figure A.3-(6)), the user may visualize these $18 \mathrm{SA}$ slices as well as the LV edge detected for that given slice and gate (Figure A.3-(7)). The screen now shows both phase and amplitude polar maps, and indicates calculated parameter values. By clicking on the Plots tab, the phase histogram is displayed on the bottom right, while on the bottom left, a radial projection curve (normalized activity as a function of radial distance) is shown in blue with a 
corresponding smoothed version shown in red and a yellow square indicating where the edge was found on the plot. The user can visualize all possible radial projections calculated by the program to determine whether the algorithms executed accurately.

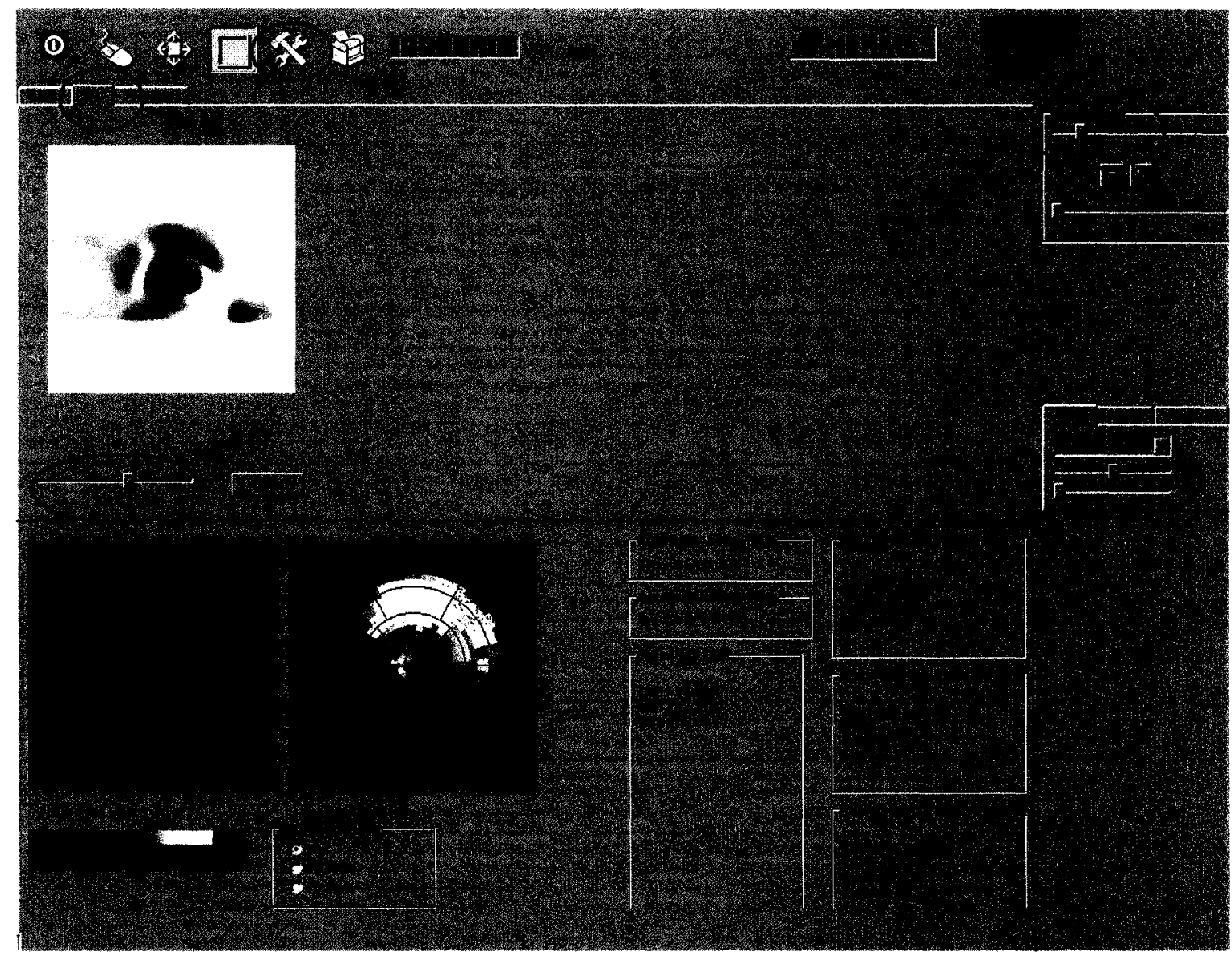

Figure A.3: Screenshot of an LV edge ROI as calculated by the program, with resulting polar maps and parameter values 


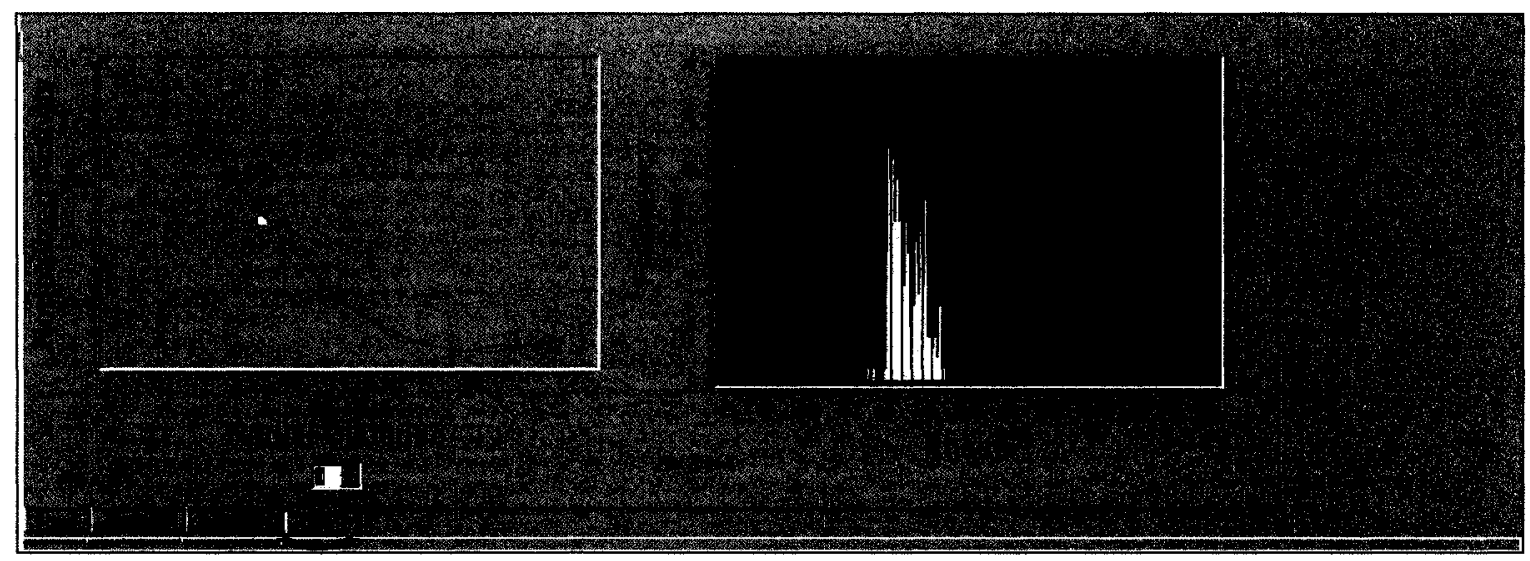

Figure A.4: Screenshot of a radial projection curve (left) and phase histogram (right). 


\section{Appendix B: Receiver Operator Characteristic Analysis}

A receiver operator characteristic (ROC) graph displays a parameter's sensitivity as a function of 1-specificity for a variety of cutoff values. Sensitivity is defined as the ratio of the positive results that the parameter determined over the actual number of positive results. Similarly, specificity is defined as the ratio of negative results that the parameter determined over the actual amount of negative results. Ideally, a test will have perfect sensitivity and perfect specificity, indicating perfect diagnostic accuracy. Equivalently, a type I error rate corresponds to 1-specificity and represents the number of false positives results over the actual number of negatives. A type II error rate represents the number of false negatives over the total number of positives. Type II error is complementary to sensitivity.

ROC curves are generated by calculating the sensitivity and specificity values of the parameter of interest at various cutoff values (Figure B.1a). For example, in this thesis, an ROC curve quantifying the ability of synchrony to detect mechanical dyssynchrony was created. Synchrony values were calculated for all subjects. In order to determine sensitivity and specificity values, a gold standard based on QRS duration and ejection fraction was used to determine the actual positive (mechanical dyssynchrony patients) and actual negatives (normal patients) test results. A cutoff value for synchrony 
was chosen and sensitivity and specificity values were calculated. Positive test results were those with synchrony values under the cutoff. A new cutoff was then chosen, in order to determine new sensitivity and specificity values. Each point on the ROC curve (Figure B.1a) therefore represents a different cutoff value of synchrony. This data was then fit to a binomial distribution. It was then possible to determine for which cutoff value the parameter performs best (i.e. desirable combination of sensitivity and specificity). For this thesis, the cutoff value, where sensitivity and specificity were approximately equal was chosen as a sample result to give an indication of both sensitivity and specificity values as well as cutoff value (Figure B.2). The area under the ROC curve was also used to determine the predictive value of the parameter. An area of 1 indicates perfect separation between data sets.
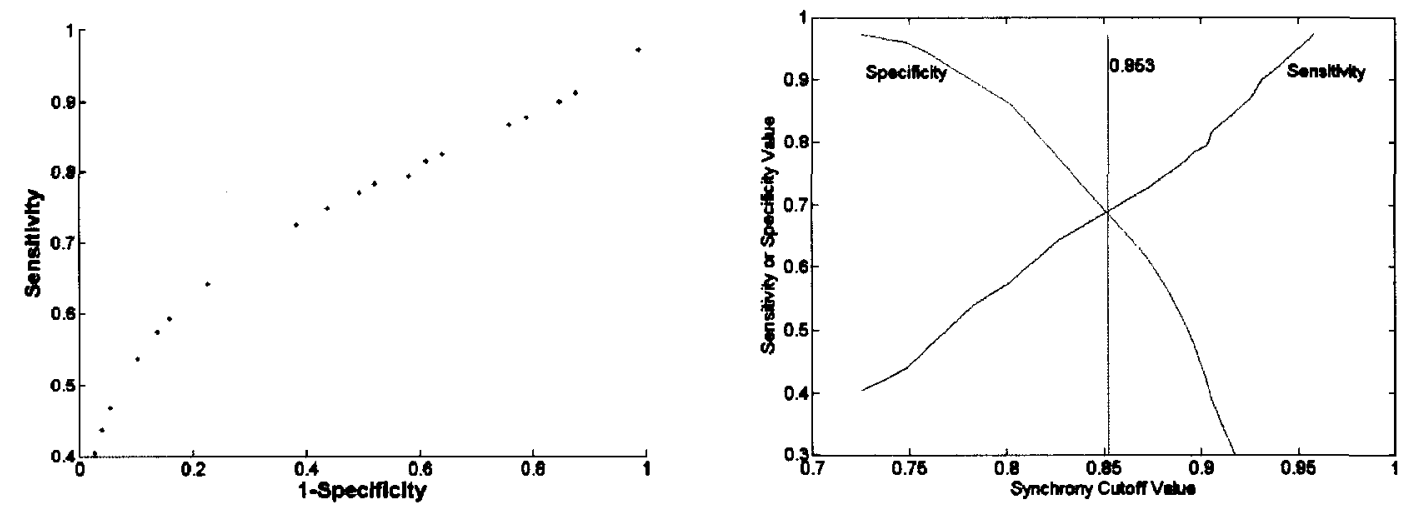

Figure B.1: a) Sample synchrony ROC curve which plots sensitivity as a function of 1specificity, and b) sensitivity (red) and specificity (blue) are plotted as a function of various synchrony cutoff values. The green line represents the location of the intersection between the two curves, and indicates cutoff value where sensitivity and specificity are equal. 


\section{References}

(1) Birnie DH, Tang AS. The problem of non-response to cardiac resynchronization therapy. Curr Opin Cardiol 2006 January;21(1):20-6.

(2) The Changing Face of Heart Disease and Stroke in Canada. Ottawa, Canada: Heart and Stroke Foundation of Canada; 1999.

(3) Statistics Canada, data transformation by Laboratory Centre for Disease Control, Health Canada. 2002.

(4) Canadian Institute for Health Information, data transormations by Laboratory Centre for Disease Control,Health Canada. 2002.

(5) Baur LH. Gated blood pool SPECT: a new clinical tool to detect cardiac dyssynchrony? Int J Cardiovasc Imaging 2008 April 24.

(6) Chen J, Henneman MM, Trimble MA et al. Assessment of left ventricular mechanical dyssynchrony by phase analysis of ECG-gated SPECT myocardial perfusion imaging. J Nucl Cardiol 2008 January;15(1):127-36.

(7) Chung ES, Leon AR, Tavazzi L et al. Results of the Predictors of Response to CRT (PROSPECT) trial. Circulation 2008 May 20;117(20):2608-16.

(8) Johansen H, Thillaiampalam S, Nguyen D, Sambell C. Diseases of the circulatory system--hospitalization and mortality. Health Rep 2005 November;17(1):49-53.

(9) Lee DS, Johansen H, Gong Y et al. Regional outcomes of heart failure in Canada. Can J Cardiol 2004 May 1;20(6):599-607.

(10) Botvinick EH. Scintigraphic blood pool and phase image analysis: the optimal tool for the evaluation of resynchronization therapy. J Nucl Cardiol 2003 July;10(4):424-8.

(11) Aehlert BH. ACLS Study Guide. Third ed. Mosby Jems Elsevier; 2007. 
(12) Penicka M, Bartunek J, De BB et al. Improvement of left ventricular function after cardiac resynchronization therapy is predicted by tissue Doppler imaging echocardiography. Circulation 2004 March 2;109(8):978-83.

(13) Toussaint JF, Lavergne T, Kerrou K et al. Basal asynchrony and resynchronization with biventricular pacing predict long-term improvement of LV function in heart failure patients. Pacing Clin Electrophysiol 2003 September;26(9):1815-23.

(14) Kapetanakis S, Kearney MT, Siva A et al. Real-time three-dimensional echocardiography: a novel technique to quantify global left ventricular mechanical dyssynchrony. Circulation 2005 August 16;112(7):992-1000.

(15) Somsen GA, Verberne HJ, Burri $\mathrm{H}$ et al. Ventricular mechanical dyssynchrony and resynchronization therapy in heart failure: a new indication for Fourier analysis of gated blood-pool radionuclide ventriculography. Nucl Med Commun 2006 February;27(2):105-12.

(16) Breithardt OA, Claus P, Sutherland GR. Do we understand who benefits from resynchronisation therapy? Eur Heart J 2004 April;25(7):535-6.

(17) Lardo AC, Abraham TP, Kass DA. Magnetic resonance imaging assessment of ventricular dyssynchrony: current and emerging concepts. J Am Coll Cardiol 2005 December 20;46(12):2223-8.

(18) Chen J, Garcia EV, Folks RD et al. Onset of left ventricular mechanical contraction as determined by phase analysis of ECG-gated myocardial perfusion SPECT imaging: development of a diagnostic tool for assessment of cardiac mechanical dyssynchrony. J Nucl Cardiol 2005 November;12(6):687-95.

(19) Ajmone MN, Henneman MM, Chen J et al. Left ventricular dyssynchrony assessed by two three-dimensional imaging modalities: phase analysis of gated myocardial perfusion SPECT and tri-plane tissue Doppler imaging. Eur J Nucl Med Mol Imaging 2008 January;35(1):166-73.

(20) Hurst JW, Morris DC, Alexander RW. The use of the New York Heart Association's classification of cardiovascular disease as part of the patient's complete Problem List. Clin Cardiol 1999 June;22(6):385-90.

(21) Butter C, Auricchio A, Stellbrink C et al. Effect of resynchronization therapy stimulation site on the systolic function of heart failure patients. Circulation 2001 December 18;104(25):3026-9. 
(22) Bleeker GB, Kaandorp TA, Lamb HJ et al. Effect of posterolateral scar tissue on clinical and echocardiographic improvement after cardiac resynchronization therapy. Circulation 2006 February 21;113(7):969-76.

(23) Towbin JA. Scarring in the heart--a reversible phenomenon? N Engl J Med 2007 October 25;357(17):1767-8.

(24) Henneman MM, Chen J, Ypenburg C et al. Phase analysis of gated myocardial perfusion single-photon emission computed tomography compared with tissue Doppler imaging for the assessment of left ventricular dyssynchrony. J Am Coll Cardiol 2007 April 24;49(16):1708-14.

(25) Yu CM, Zhang Q, Chan YS et al. Tissue Doppler velocity is superior to displacement and strain mapping in predicting left ventricular reverse remodelling response after cardiac resynchronisation therapy. Heart 2006 October;92(10):1452-6.

(26) Fauchier L, Marie O, Casset-Senon D et al. Reliability of QRS duration and morphology on surface electrocardiogram to identify ventricular dyssynchrony in patients with idiopathic dilated cardiomyopathy. Am J Cardiol 2003 August 1;92(3):341-4.

(27) Yu CM, Abraham WT, Bax J et al. Predictors of response to cardiac resynchronization therapy (PROSPECT)--study design. Am Heart J 2005 April;149(4):600-5.

(28) Westenberg JJ, Lamb HJ, van der Geest RJ et al. Assessment of left ventricular dyssynchrony in patients with conduction delay and idiopathic dilated cardiomyopathy: head-to-head comparison between tissue doppler imaging and velocity-encoded magnetic resonance imaging. J Am Coll Cardiol 2006 May 16;47(10):2042-8.

(29) White JA, Yee R, Yuan X et al. Delayed enhancement magnetic resonance imaging predicts response to cardiac resynchronization therapy in patients with intraventricular dyssynchrony. J Am Coll Cardiol 2006 November 21;48(10):1953-60.

(30) Smith WH, Kastner RJ, Calnon DA et al. Quantitative gated single photon emission computed tomography imaging: a counts-based method for display and measurement of regional and global ventricular systolic function. J Nucl Cardiol 1997 November;4(6):451-63.

(31) Calnon DA, Kastner RJ, Smith WH et al. Validation of a new counts-based gated single photon emission computed tomography method for quantifying left 
ventricular systolic function: comparison with equilibrium radionuclide angiography. J Nucl Cardiol 1997 November;4(6):464-71.

(32) Henneman MM, Chen J, Gibbets-Schneider P et al. Can LV dyssynchrony as assessed with phase analysis on gated myocardial perfusion SPECT predict response to CRT? J Nucl Med 2007 July;48(7):1104-11.

(33) Harel F, Finnerty V, Ngo Q et al. SPECT versus planar gated blood pool imaging for left ventricular evaluation. J Nucl Cardiol 2007 July;14(4):544-9.

(34) Adachi I, Akagi H, Umeda T et al. Gated blood pool SPECT improves reproducibility of right and left ventricular Fourier phase analysis in radionuclide angiography. Ann Nucl Med 2003 December;17(8):711-6.

(35) O'Connell JW, Schreck C, Moles M et al. A unique method by which to quantitate synchrony with equilibrium radionuclide angiography. J Nucl Cardiol 2005 July;12(4):441-50.

(36) Nuclear Decay Data in the MIRD Format. National Nuclear Data Center 2008; Available from: URL: http://www.nndc.bnl.gov/mird

(37) Fernow RC. Introduction to experimental particle physics. Cambridge, Cambridgeshire: Cambridge University Press; 1986.

(38) Prince JL, Links JM. Medical Imaging Signals and Systems. Pearson Prentice Hall; 2006.

(39) Links JM, Douglass KH, Wagner HN, Jr. Patterns of ventricular emptying by Fourier analysis of gated blood-pool studies. J Nucl Med 1980 October;21(10):978-82.

(40) Siegrist PT, Comte N, Holzmeister J et al. Effects of AV delay programming on ventricular resynchronisation: role of radionuclide ventriculography. Eur J Nucl Med Mol Imaging 2008 March 19.

(41) Fauchier L, Marie O, Casset-Senon D et al. Interventricular and intraventricular dyssynchrony in idiopathic dilated cardiomyopathy: a prognostic study with fourier phase analysis of radionuclide angioscintigraphy. J Am Coll Cardiol 2002 December 4;40(11):2022-30.

(42) Harel F, Finnerty V, Gregoire J et al. Comparison of left ventricular contraction homogeneity index using SPECT gated blood pool imaging and planar phase analysis. J Nucl Cardiol 2008 January;15(1):80-5. 
(43) Casset-Senon D, Babuty D, Philippe L et al. Fourier phase analysis of SPECT equilibrium radionuclide angiography in symptomatic patients with mitral valve prolapse without significant mitral regurgitation: assessment of biventricular functional abnormalities suggesting a cardiomyopathy. J Nucl Cardiol 2000 September;7(5):471-7.

(44) Muramatsu T, Matsumoto K, Nishimura S. Efficacy of the phase images in Fourier analysis using gated cardiac POOL-SPECT for determining the indication for cardiac resynchronization therapy. Circ J 2005 December;69(12):1521-6.

(45) Frais M, Botvinick E, Shosa D et al. Phase image characterization of localized and generalized left ventricular contraction abnormalities. J Am Coll Cardiol 1984 November;4(5):987-98.

(46) Cerqueira MD, Weissman NJ, Dilsizian V et al. Standardized myocardial segmentation and nomenclature for tomographic imaging of the heart. A statement for healthcare professionals from the Cardiac Imaging Committee of the Council on Clinical Cardiology of the American Heart Association. Int J Cardiovasc Imaging 2002 February;18(1):539-42.

(47) Song ZZ. Can LV dyssynchrony as assessed with phase analysis on gated myocardial perfusion SPECT preferably predict response to CRT? J Nucl Med 2008 April;49(4):686.

(48) Dinu C, Klein G, Morestin-Cadet S et al. Gated blood pool tomoscintigraphy with 4-dimensional optical flow motion analysis quantifies left ventricular mechanical activation and synchronization. J Nucl Cardiol 2006 November;13(6):811-20. 